\title{
Robust optimal experiment design for system identification ${ }^{\text {is }}$
}

\author{
Cristian R. Rojas ${ }^{\text {a }}$, James S. Welsh ${ }^{\mathrm{a}, *}$, Graham C. Goodwin ${ }^{\mathrm{a}}$, Arie Feuer ${ }^{\mathrm{b}}$ \\ ${ }^{\mathrm{a}}$ School of Electrical Engineering and Computer Science, The University of Newcastle, Callaghan, NSW 2308, Australia \\ ${ }^{\mathrm{b}}$ Department of Electrical Engineering, Technion - Israel Institute of Technology, Haifa 32000, Israel
}

Received 27 November 2005; received in revised form 3 August 2006; accepted 4 December 2006

\begin{abstract}
This paper develops the idea of min-max robust experiment design for dynamic system identification. The idea of min-max experiment design has been explored in the statistics literature. However, the technique is virtually unknown by the engineering community and, accordingly, there has been little prior work on examining its properties when applied to dynamic system identification. This paper initiates an exploration of these ideas. The paper considers linear systems with energy (or power) bounded inputs. We assume that the parameters lie in a given compact set and optimise the worst case over this set. We also provide a detailed analysis of the solution for an illustrative one parameter example and propose a convex optimisation algorithm that can be applied more generally to a discretised approximation to the design problem. We also examine the role played by different design criteria and present a simulation example illustrating the merits of the proposed approach.
\end{abstract}

(C) 2007 Elsevier Ltd. All rights reserved.

Keywords: Robust experiment design; Optimal input design

\section{Introduction}

The goal of experiment design is to adjust the experimental conditions so that maximal information is gained from the experiment. Background to this problem can be found in early statistics literature (Cox, 1958; Fedorov, 1972; Karlin \& Studden, 1966; Kempthorne, 1952; Kiefer \& Wolfowitz, 1960; Wald, 1943; Whittle, 1973; Wynn, 1972) as well as in the engineering literature (Arimoto \& Kimura, 1973; Gagliardi, 1967; Goodwin, Murdoch, \& Payne, 1973; Goodwin \& Payne, 1973; Goodwin, Payne, \& Murdoch, 1973; Goodwin \& Payne, 1977; Hildebrand \& Gevers, 2003a; Levadi, 1966; Mehra, 1974; Zarrop, 1979). A recent survey is contained in Gevers (2005) where many additional references can be found. The focus in the engineering literature has been predominately on experiment design for dynamic system identification.

A key issue with experiment design for dynamic systems is that the model is typically nonlinearly parameterised. This

\footnotetext{
This paper was not presented at any IFAC meeting. This paper was recommended for publication in revised form by Associate Editor Johan Schoukens under the direction of Editor Torsten Söderström.

* Tel.: +61 249216087 ; fax: +61 249216993.

E-mail address: james.welsh@newcastle.edu.au (J.S. Welsh).
}

means, amongst other things, that the Fisher information matrix (Goodwin \& Payne, 1977, p. 6) which is typically used as the basis for experiment design, depends, inter alia, on the true system parameters (i.e. the nominal optimal experiment depends on the very thing that the experiment is aimed at finding).

This issue has been recognised in the statistics literature where several approaches have been explored. These include:

- Sequential design, where one iterates between parameter estimation, on the one hand, and experiment design using the current parameter estimates, on the other, see Chernoff (1975), Ford and Silvey (1980), Ford, Titterington, and Wu (1985), Müller and Pötscher (1992), Walter and Pronzato (1997), and Wu (1985).

- Bayesian design (Atkinson, Chaloner, Juritz, \& Herzberg, 1993; Atkinson \& Doner, 1992; Chaloner \& Larntz, 1989; Chaloner \& Verdinelli, 1995; El-Gamal \& Palfrey, 1996; Sebastiani \& Wynn, 2000). The Bayesian approach is characterised by the minimisation of the expected value (over the prior parameter distribution) of a local optimality criterion related to the information matrix.

- Min-max design (Biedermann \& Dette, 2003; D'Argenio \& Van Guilder, 1988; Dette, Melas, \& Pepelyshev, 2003; Fedorov, 1980; Landaw, 1984; Melas, 1978; Pronzato \& Walter, 1988). 
However, there has been little work on robust experiment design for engineering problems. This has been highlighted in the recent survey paper (Hjalmarsson, 2005, p. 427) where it is stated that "...as usual in experiment design, in order to compute the optimal design the true system has to be known. Methods that are robust with respect to uncertainty about the system is a wide open research field."

Preliminary work in the engineering literature on robust experiment design includes substantial work on iterative design (Gevers, 2005; Hjalmarsson, 2005) and an insightful suboptimal min-max solution for a one parameter problem (Walter \& Pronzato, 1997, p. 339). Actually the latter problem will be discussed in detail in Section 3 of the current paper. Also, a number of very recent engineering papers refer to the idea of min-max optimal experiment design-see for example papers presented at SYSID'06, e.g., Gevers and Bombois (2006), Goodwin, Welsh, Feuer, and Derpich (2006), and Mårtensson and Hjalmarsson (2006).

Our goal in the current paper is to develop the idea of min-max optimal experiment design for dynamic system identification. To gain insight into this approach, we explore an illustrative example in depth.

We assume prior knowledge in the form that the system parameters, $\theta$, are contained in a given compact set $\Theta$. We then choose a design criterion $f(M(\theta), \theta)$ where $M(\theta)$ is the Fisher information matrix, evaluated at $\theta$, and design the experiment to optimise the worst case of $f(M(\theta), \theta)$ over $\Theta$. Notice that this differs from the usual approaches to experiment design in the engineering literature which typically optimise $f\left(M\left(\theta_{0}\right), \theta_{0}\right)$ for some given nominal value $\theta_{0}$.

Our approach is more akin to the usual formulation of robust optimal control which typically considers the worst case (Zhou, Doyle, \& Glover, 1996). Indeed, there are substantial links between the work presented here and continuous game theory (Başar \& Bernhard, 1995; Başar \& Olsder, 1995; Fudenberg \& Tirole, 1991; Owen, 1995; Szép \& Forgó, 1985). We explore some of these connections below.

The merits of the approach proposed in this paper are illustrated by an example (presented in Section 5) which shows, for a realistic second order system, that an order of magnitude improvement in the worst case performance in experiment design can be achieved at the expense of only a few percent degradation in the nominal performance.

The layout of the remainder of the paper is as follows: in Section 2 we give a general formulation of the min-max approach to robust optimal experiment design. Section 3 explores an illustrative one parameter example in considerable detail so as to give insight into the problem. In Section 4 we describe the extension to multi-parameter systems. In Section 5 we present several results illustrating the merits of the proposed approach. Finally, in Section 6 we draw conclusions.

\section{Experiment design criteria}

\subsection{The information matrix}

So as to be specific we first consider a single input single output linear discrete time system, with input $\left\{u_{t}\right\}$ and output $\left\{y_{t}\right\}$, of the form

$y_{t}=G_{1}(q) u_{t}+G_{2}(q) w_{t}$,

where $G_{1}$ and $G_{2}$ are rational transfer functions, $q$ is the forward shift operator, $G_{2}(\infty)=1$, and $\left\{w_{t}\right\}$ is zero mean Gaussian white noise of variance $\Sigma$. We let $\beta \triangleq\left[\theta^{\mathrm{T}}, \gamma^{\mathrm{T}}, \Sigma\right]^{\mathrm{T}}$ where $\theta$ denotes the parameters in $G_{1}$ and $\gamma$ denotes the parameters in $G_{2}$.

We recall that the log likelihood function (Goodwin \& Payne, 1977, p. 130) for data $Y$ given parameters $\beta$, is

$\ln p(Y \mid \beta)=-\frac{N}{2} \ln 2 \pi-\frac{N}{2} \ln \Sigma-\frac{1}{2 \Sigma} \sum_{t=1}^{N} \varepsilon_{t}^{2}$,

where

$\varepsilon_{t} \triangleq G_{2}(q)^{-1}\left[y_{t}-G_{1}(q) u_{t}\right]$.

Fisher's information matrix is obtained by taking the following expectation (Goodwin \& Payne, 1977, p. 130):

$M \triangleq \mathbb{E}_{Y \mid \beta}\left[\left(\frac{\partial \ln p(Y \mid \beta)}{\partial \beta}\right)\left(\frac{\partial \ln p(Y \mid \beta)}{\partial \beta}\right)^{T}\right]$,

where from (1)

$$
\begin{aligned}
& \frac{\partial \ln p(Y \mid \beta)}{\partial \beta} \\
& =-\frac{1}{\Sigma} \sum_{t=1}^{N} \varepsilon_{t} \frac{\partial \varepsilon_{t}}{\partial \beta}-\frac{1}{2 \Sigma} \frac{\partial \Sigma}{\partial \beta}\left[N-\frac{1}{\Sigma} \sum_{t=1}^{N} \varepsilon_{t}^{2}\right],
\end{aligned}
$$

from (2)

$$
\frac{\partial \varepsilon_{t}}{\partial \beta}=-G_{2}(q)^{-1}\left\{\frac{\partial G_{2}(q)}{\partial \beta} \varepsilon_{t}+\frac{\partial G_{1}(q)}{\partial \beta} u_{t}\right\}
$$

and where $\mathbb{E}_{Y \mid \beta}$ denotes the expectation over the distribution of the data given $\beta$.

We assume an open-loop experiment so that $w_{t}$ and $u_{t}$ are uncorrelated. We also assume that $G_{1}, G_{2}$ and $\Sigma$ are independently parameterised. Taking expectations, as in (3), $M$ can be partitioned as

$M=\left[\begin{array}{cc}M_{1} & 0 \\ 0 & M_{2}\end{array}\right]$

where $M_{1}$ is the part of the information matrix related to $\theta$, and $M_{2}$ is independent of the input. Thus,

$M_{1} \triangleq \frac{1}{\Sigma} \sum_{t=1}^{N}\left(\frac{\partial \varepsilon_{t}}{\partial \theta}\right)\left(\frac{\partial \varepsilon_{t}}{\partial \theta}\right)^{T}$,

where $\partial \varepsilon_{t} / \partial \theta$ satisfies

$\frac{\partial \varepsilon_{t}}{\partial \theta}=-G_{2}(q)^{-1} \frac{\partial G_{1}(q)}{\partial \theta} u_{t}$.

Notice that $M_{1}$ depends on the full parameter vector $\beta$. Assuming $N$ is large, it is more convenient to work with the scaled 
average information matrix for the parameters $\theta$ (Goodwin \& Payne, 1977, p. 134; Walter \& Pronzato, 1997, p. 208),

$\bar{M}\left(\beta, \phi_{u}\right) \triangleq \lim _{N \rightarrow \infty} \frac{1}{N} M_{1} \Sigma$.

Utilising Parseval's Theorem, we finally have that

$\bar{M}\left(\beta, \phi_{u}\right)=\frac{1}{\pi} \int_{0}^{\pi} \tilde{M}(\beta, \omega) \phi_{u}\left(\mathrm{e}^{\mathrm{j} \omega}\right) \mathrm{d} \omega$,

where

$\tilde{M}(\beta, \omega) \triangleq \operatorname{Re}\left\{\frac{\partial G_{1}\left(\mathrm{e}^{\mathrm{j} \omega}\right)}{\partial \theta}\left|G_{2}\left(\mathrm{e}^{\mathrm{j} \omega}\right)\right|^{-2}\left[\frac{\partial G_{1}\left(\mathrm{e}^{\mathrm{j} \omega}\right)}{\partial \theta}\right]^{H}\right\}$

and $\phi_{u}$ is the discrete time input spectral density (considered as a generalised function). Here, $H$ is the conjugate transpose operator.

It is also possible to do a parallel development (Goodwin \& Payne, 1977, p. 142) for continuous time models. In the latter case, (5) is replaced by

$\bar{M}\left(\beta, \phi_{u}\right)=\int_{0}^{\infty} \tilde{M}(\beta, \omega) \phi_{u}(\omega) \mathrm{d} \omega$,

where

$\tilde{M}(\beta, \omega) \triangleq \operatorname{Re}\left\{\frac{\partial G_{1}(\mathrm{j} \omega)}{\partial \theta}\left|G_{2}(\mathrm{j} \omega)\right|^{-2}\left[\frac{\partial G_{1}(\mathrm{j} \omega)}{\partial \theta}\right]^{H}\right\}$,

where $G_{1}$ and $G_{2}$ are continuous time transfer functions (assumed independently parameterised) and $\phi_{u}$ is the continuous time input spectral density.

Notice that the results presented below do not depend on $\Sigma$ since it appears as a scaling factor in (4). Also, we see from (6) that, in $\bar{M}\left(\beta, \phi_{u}\right), G_{2}$ simply plays the role of a frequency dependent weighting. This is easily included in the analysis. However, for simplicity we assume white noise, although the extension to nonwhite noise is straightforward. Hence in the sequel we refer only to $\theta$.

\subsection{Brief review of design criteria for nominal experiment design}

Since $\bar{M}$ is a matrix, we need a scalar measure of $\bar{M}$ for the purpose of experiment design. In the nominal case typically treated in the engineering literature (i.e. when a fixed prior estimate of $\theta$ is used), several measures of the "size" of $\bar{M}$ have been proposed. Some examples are:

(i) D-optimality (Goodwin \& Payne, 1977, p. 126)

$$
J_{d}\left(\theta, \phi_{u}\right) \triangleq\left[\operatorname{det} \bar{M}\left(\theta, \phi_{u}\right)\right]^{-1} .
$$

(ii) Experiment design for robust control (Hildebrand \& Gevers, 2003a, 2003b; Hjalmarsson, 2005, p. 427).

$$
J_{r c}\left(\theta, \phi_{u}\right) \triangleq \sup _{\omega} g(\theta, \omega)^{\mathrm{H}} \bar{M}^{-1} g(\theta, \omega),
$$

where $g$ is a frequency dependent vector related to the $v$ gap (Hildebrand \& Gevers, 2003a, 2003b).

Many other criteria have been described in the statistics literature, such as $A$-optimality $\left(\operatorname{tr} \bar{M}\left(\theta, \phi_{u}\right)^{-1}\right), L$ optimality $\left(\operatorname{tr} W \bar{M}\left(\theta, \phi_{u}\right)^{-1}\right.$, for some $\left.W \geqslant 0\right)$ and $E$-optimality $\left(\lambda_{\max }\left(\bar{M}\left(\theta, \phi_{u}\right)^{-1}\right)\right)$; see Kiefer (1974). On the other hand, in the engineering literature, (Bombois, Scorletti, Gevers, Van den Hof, \& Hildebrand, 2005), for example, proposed a criterion that leads to the required accuracy to achieve a given level of robust control performance. Other criteria will be discussed in Section 4.

A common feature of all these nominal experiment design approaches is that they are aimed at choosing $\phi_{u}$ to minimise a function of the type shown in (7) and (8). Notice, however, that the optimal input spectrum depends, inter-alia, on the unknown parameter vector $\theta$.

\subsection{Min-max robust design}

A min-max robust design criterion is the basis of the approach described in the current paper. Specifically, we assume that we have available a priori information showing that the parameters can take any value in a compact set $\Theta$. We also constrain the allowable set of input signals. A typical constraint (Goodwin \& Payne, 1977, p. 133; Walter \& Pronzato, 1997, p. 308; Zarrop, 1979, p. 26) used in experiment design is that the input energy is constrained, i.e. we define ${ }^{1}$

$\mathscr{S}\left(\mathbb{R}_{0}^{+}\right) \triangleq\left\{\phi_{u}: \mathbb{R} \rightarrow \mathbb{R}_{0}^{+}: \quad \begin{array}{c}\operatorname{supp} \phi_{u} \subset \mathbb{R}_{0}^{+} \quad \text { and } \\ \int_{-\infty}^{\infty} \phi_{u}(\omega) \mathrm{d} \omega=1\end{array}\right\}$.

The min-max robust optimal input spectral density, $\phi_{u}^{*}$, is then chosen as

$\phi_{u}^{*}=\arg \min _{\phi_{u} \in \mathscr{S}\left(\mathbb{R}_{0}^{+}\right)} \sup _{\theta \in \Theta} J\left(\bar{M}\left(\theta, \phi_{u}\right), \theta\right)$,

where $J$ is an appropriate scalar measure of $\bar{M}$. We are assuming for the moment that $\phi_{u}^{*}$ exists and is unique; these points will be studied in the next section. Notice also that we allow $J$ to depend explicitly on $\theta$; this point will be of practical importance-see discussion below.

\subsection{A mixed policy game approach}

An alternative approach to that described above would be to extend the space to include "mixed policies" (Başar \& Bernhard, 1995, p. 35) by introducing a (generalised probability) density $\xi$ on $\Theta$, i.e. $\xi \in \mathscr{S}(\Theta)$. The counterpart of (9) would now take the form

$\phi_{u}^{*}=\arg \min _{\phi_{u} \in \mathscr{S}\left(\mathbb{R}_{0}^{+}\right)} \sup _{\xi \in \mathscr{S}(\Theta)} J^{\prime}\left(\xi, \phi_{u}\right)$,

\footnotetext{
${ }^{1}$ In general, given a set $X \subset \mathbb{R}^{n}$, we will denote by $\mathscr{S}(X)$ the set of all generalised functions $\phi_{u}$ on $\mathbb{R}^{n}$ (Rudin, 1973, Chapter 6) such that $\phi_{u}$ is the derivative of some probability distribution function on $\mathbb{R}^{n}$, and supp $\phi_{u} \subset X$, where supp $\phi_{u}$ is the support of $\phi_{u}$ (i.e. roughly speaking, $\mathscr{S}(X)$ is the set of all (generalised) probability density functions on $X$ ).
} 
where $J^{\prime}$ is an appropriate scalar measure of the form

$J^{\prime} \triangleq \int f\left(\int S_{\theta} \tilde{M}(\theta, \omega) S_{\theta}^{T} \phi_{u}(\omega) \mathrm{d} \omega\right) \xi(\theta) \mathrm{d} \theta$,

where $f$ is a scalar-valued function, e.g. $f(L)=\operatorname{tr} L^{-1}$ or $f(L)=$ $\lambda_{\max }\left(L^{-1}\right) ; \widetilde{M}$ is the single frequency information matrix and $S_{\theta}$ is a parameter dependent scaling matrix (see Section 4.1 for further discussion of $S_{\theta}$ ).

Notice that if $f$ were linear, it could be introduced into the inner integral, and in that case it can be shown that this approach is equivalent to the previous one (see the proof of Theorem 3 for an idea of how the equivalence is established).

\section{An illustrative example}

Before delving into the general multi-parameter case, we first consider an illustrative continuous time one parameter problem to gain insight. We take $G_{2}(s)=1$ and let

$G_{1}(s)=\frac{1}{s / \theta+1}$.

Notice that this problem has also been discussed in Goodwin and Payne (1977, p. 142) for the case of nominal experiment design. Also, the problem has been studied in Walter and Pronzato (1997, p. 339) in the context of min-max robust experiment design but where the input is restricted to a single sinusoid. Actually we will see below that the later restriction unduely limits the solution space and does not lead to the optimal strategy when $\theta \in[\underline{\theta}, \bar{\theta}]$, with $\bar{\theta} / \underline{\theta}>2+\sqrt{3}$ (see the Appendix). This is heuristically reasonable since if $\theta$ lies in an interval, then it makes sense to spread the input energy in some sense to cover the possible $\theta$ scenarios.

For the model (10), it follows that

$\bar{M}\left(\theta, \phi_{u}\right)=\int_{0}^{\infty} \tilde{M}(\theta, \omega) \phi_{u}(\omega) \mathrm{d} \omega$,

where $\tilde{M}$ is the "single frequency" normalised information matrix given by

$\tilde{M}(\theta, \omega)=\left|\frac{\partial G_{1}(\theta, \omega)}{\partial \theta}\right|^{2}=\frac{\omega^{2} / \theta^{4}}{\left(\omega^{2} / \theta^{2}+1\right)^{2}}$.

\subsection{Nominal optimal experiment design for the illustrative example}

Before turning to the robust design problem, we will briefly review the nominal experiment design problem for this case. Here one assumes that a prior estimate, $\widehat{\theta}$, of $\theta$ is available. Based on this information, the function $\phi_{u}$ is chosen so as to optimise some scalar-valued function of $\bar{M}\left(\widehat{\theta}, \phi_{u}\right)$ subject to a constraint on the input power. In the nominal case it can be shown that we only need to use a single frequency input for this example (Goodwin \& Payne, 1977, p. 143), namely, $\phi_{u}(\omega)=\delta\left(\omega-\omega^{*}\right)$. Moreover, by differentiation of the single frequency information matrix given in (11), it is readily seen

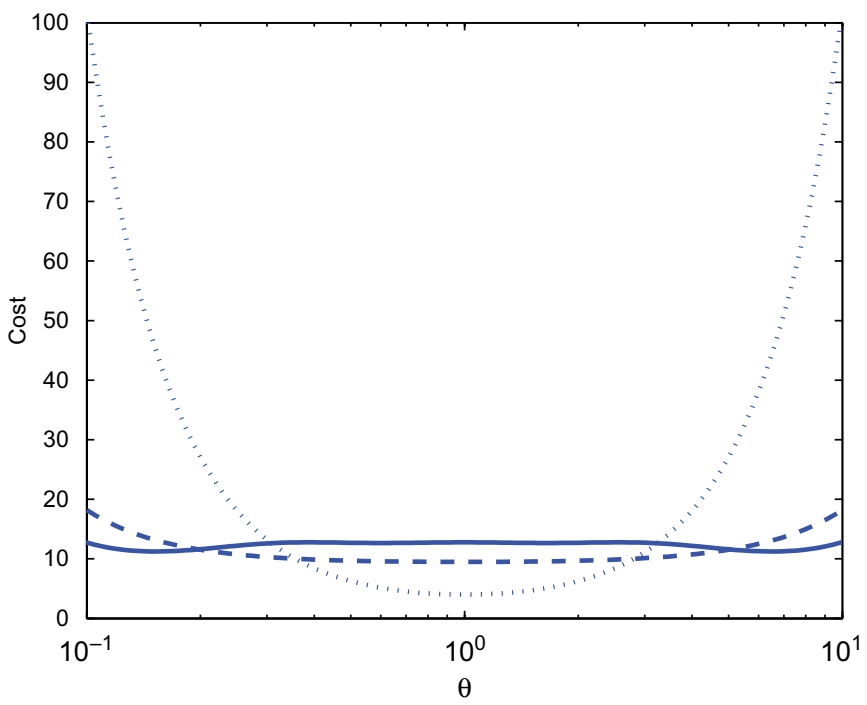

Fig. 1. $\left[\theta^{2} \bar{M}\left(\theta, \phi_{u}\right)\right]^{-1}$ as a function of $\theta$ for nominal input (dotted), robust optimal input (solid) and ' $1 / f$ ' noise (dashed).

that the optimal input frequency is

$\omega^{*}=\theta$.

This is an intuitively pleasing result, i.e. one places the test signal at the (nominal) $3 \mathrm{~dB}$ break point. However, Eq. (12) reinforces the fundamental difficulty in nominal experiment design, namely, the optimal experiment depends on the very thing that the experiment is aimed at estimating.

To gauge the importance of the dependence on $\theta$, we notice that $\widetilde{M}(\theta, \omega)$ in our example decays at the rate of $40 \mathrm{~dB}$ per decade as a function of both $\theta$ and $\omega$. Hence, given the prior estimate of the parameter, $\widehat{\theta}$, say we choose $\omega^{*}=\widehat{\theta}$ for the input signal frequency. Also, say that the true parameter lies in the range $(0.1 \widehat{\theta} \leqslant \theta \leqslant 10 \widehat{\theta})$, then $\min _{\theta \in \Theta} \widetilde{M}(\theta, \omega)$ is approximately $\frac{1}{100}$ th of the nominal value! This suggests that nominal experiment design is limited to those cases where an extremely good prior estimate is available. This point is reinforced in Fig. 1 which shows a plot of $\left[\theta^{2} \bar{M}\left(\theta, \phi_{u}\right)\right]^{-1}$ versus $\theta$ for the nominal optimal input.

Remark 1. The reason for multiplying $\bar{M}\left(\theta, \phi_{u}\right)$ by $\theta^{2}$, and then inverting, as in Fig. 1, is that $\bar{M}^{-1}$ is a variance measure and thus $\left[\theta^{2} \bar{M}\right]^{-1}$ gives relative (mean square) errors. More will be said about this type of scaled cost function in the context of robust design below. Interestingly, the scaling turns out to be equivalent (for this example) to the MMDE cost function used in (Walter \& Pronzato, 1997, p. 339).

\subsection{Some properties of the min-max robust optimal experiment design}

We next turn to robust experiment design as described in Section 2. For the illustrative problem we use (see Remark 1 and Section 4)

$J\left(\bar{M}\left(\theta, \phi_{u}\right), \theta\right) \triangleq\left[\theta^{2} \bar{M}\left(\theta, \phi_{u}\right)\right]^{-1}$. 
Thus, our min-max robust optimal experiment design can be stated as finding

$\phi_{u}^{*}=\arg \min _{\phi_{u} \in \mathscr{S}\left(\mathbb{R}_{0}^{+}\right)} \bar{J}\left(\phi_{u}\right)$,

where

$\bar{J}\left(\phi_{u}\right) \triangleq \sup _{\theta \in \Theta}\left[\int_{0}^{\infty} \frac{\omega^{2} / \theta^{2}}{\left(\omega^{2} / \theta^{2}+1\right)^{2}} \phi_{u}(\omega) \mathrm{d} \omega\right]^{-1}$

and $\Theta \triangleq[\underline{\theta}, \bar{\theta}]$. In the sequel, we will give further insights into the above design problem.

We first observe that, since $\theta^{2} \tilde{M}(\theta, \omega)$ in (14) is continuous in $\theta \in \Theta$ for every $\omega \in \mathbb{R}_{0}^{+}$and it is bounded by an integrable function which is independent of $\theta$ (use e.g. $C / \omega^{2}$, where $C$ is large and independent of $\theta$ ), the integral is continuous in $\theta$; see Bartle (1966, p. 46). This implies, with the compactness of $\Theta$, that we can change "sup" in (14) to "max".

Furthermore, if we make the following change of variables:

$x \triangleq \frac{\ln \theta-\ln \underline{\theta}}{\ln \bar{\theta}-\ln \underline{\theta}}$

$y \triangleq \frac{\ln \omega-\ln \underline{\theta}}{\ln \bar{\theta}-\ln \underline{\theta}}$

$\phi_{u}(\omega)=\frac{2}{k \omega} \widetilde{\phi}_{u}\left(\frac{\ln \omega-\ln \underline{\theta}}{\ln \bar{\theta}-\ln \underline{\theta}}\right)$,

$k \triangleq 2(\ln \bar{\theta}-\ln \underline{\theta})$

then the problem can be rewritten as

$\widetilde{\phi}_{u}^{*}=\arg \max _{\widetilde{\phi}_{u} \in \mathscr{S}(\mathbb{R})} \min _{x \in[0,1]} \int_{-\infty}^{\infty} \frac{\mathrm{e}^{k(x-y)}}{\left(\mathrm{e}^{k(x-y)}+1\right)^{2}} \widetilde{\phi}_{u}(y) \mathrm{d} y$.

To simplify the notation, let $F(x, y) \triangleq f(x-y)$, where $f(u) \triangleq \mathrm{e}^{k u} /\left(\mathrm{e}^{k u}+1\right)^{2}$.

The following theorems give some properties of $\phi_{u}^{*}$ and $\widetilde{\phi}_{u}^{*}$. Notice that Lemma 2 and Theorem 5 are different in that Lemma 2 states that the optimal input has compact support, which is a technical requirement for proving other results. Theorem 5, on the other hand, states that the optimal input has finite support, which is a stronger result than Lemma 2, but its proof relies on the previous theorems.

Some of the results below are based on the fact that if $f$ is a continuous function on $[a, b]$, then

$\min _{g \in \mathscr{S}([a, b])} \int_{a}^{b} f(x) g(x) \mathrm{d} x=\min _{x \in[a, b]} f(x)$.

By choosing as $g$ a Dirac delta at a point $x \in[a, b]$ for which $f(x)$ is minimum, we see that the right side of (17) is not less than its left side. The other inequality can be deduced from the Mean Value Theorem for integrals (Apostol, 1974, p. 360).

Lemma 2. Considering the problem stated in (16), the optimal input $\widetilde{\phi}_{u}^{*}$, if it exists, has all its energy inside [0, 1]. Namely,

$\int_{\mathbb{R}-[0,1]} \widetilde{\phi}_{u}^{*}(y) \mathrm{d} y=0$.
Thus, the spectral density of the optimal input has compact support, i.e. $\widetilde{\phi}_{u}^{*} \in \mathscr{S}([0,1])$ (or, equivalently, $\phi_{u}^{*} \in \mathscr{S}(\Theta)$ ), so we can replace (16) with

$\widetilde{\phi}_{u}^{*}=\arg \max _{\widetilde{\phi}_{u} \in \mathscr{S}([0,1])} \min _{x \in[0,1]} \int_{0}^{1} \frac{\mathrm{e}^{k(x-y)}}{\left(\mathrm{e}^{k(x-y)}+1\right)^{2}} \widetilde{\phi}_{u}(y) \mathrm{d} y$.

Proof. Notice ${ }^{2}$ that $\partial F / \partial y>0$ for $y<x$ and $\partial F / \partial y<0$ for $y>x$. It follows that for any $x \in[0,1]$ we have $\int_{-\infty}^{\infty} F(x, y) \widetilde{\phi}_{u}^{*}(y) \mathrm{d} y \leqslant \int_{-\infty}^{\infty} F(x, y) \widetilde{\phi}_{u}^{\prime}(y) \mathrm{d} y$, where $\widetilde{\phi}_{u}^{\prime}$ is given by

$$
\begin{aligned}
\widetilde{\phi}_{u}^{\prime}(y) \triangleq & \widetilde{\phi}_{u}^{*}(y) \mathscr{X}_{[0,1]}(y)+\delta(y) \int_{-\infty}^{0_{-}} \widetilde{\phi}_{u}^{*}(\tau) \mathrm{d} \tau \\
& +\delta(y-1) \int_{1_{+}}^{\infty} \widetilde{\phi}_{u}^{*}(\tau) \mathrm{d} \tau
\end{aligned}
$$

and $\mathscr{X}_{[0,1]}$ is the indicator function of $[0,1]$. The result follows.

Theorem 3. For the problem stated in (13) or (16), there exists at least one optimal input, that is, there exists a $\phi_{u}^{*} \in \mathscr{S}\left(\mathbb{R}_{0}^{+}\right)$ such that for every $\phi_{u} \in \mathscr{S}\left(\mathbb{R}_{0}^{+}\right)$,

$\bar{J}\left(\phi_{u}^{*}\right) \leqslant \bar{J}\left(\phi_{u}\right)$

Proof. By Lemma 2, (16) can be related to a two-person zerosum game on the unit square with kernel $F$, such that player $x$ tries to minimise $F$ by using a pure strategy, and player $y$ tries to maximise this quantity by using a mixed strategy (Başar \& Olsder, 1995, p. 25). Hence, in order to prove the existence of $\widetilde{\phi}_{u}^{*}$ (or of $\phi_{u}^{*}$, which is the same), we can make use of a version of the Minimax Theorem, due to Glicksberg (1950), which states that if $F$ is an upper or lower semicontinuous function on $[0,1] \times[0,1]$, then

$$
\begin{aligned}
& \inf _{\mu_{x} \in \mathscr{S}([0,1])} \sup _{\mu_{y} \in \mathscr{S}([0,1])} \int_{0}^{1} \int_{0}^{1} F(x, y) \mu_{x}(x) \mu_{y}(y) \mathrm{d} y \mathrm{~d} x \\
& =\sup _{\mu_{y} \in \mathscr{S}([0,1])} \inf _{\mu_{x} \in \mathscr{S}([0,1])} \int_{0}^{1} \int_{0}^{1} F(x, y) \mu_{x}(x) \mu_{y}(y) \mathrm{d} y \mathrm{~d} x \\
& \triangleq V_{m}
\end{aligned}
$$

where $V_{m}$ is called the average value of the game. Furthermore, if $F$ is continuous then, by a standard compactness argument (such as that given in the paragraph before (15)), there exist $\mu_{x}^{*}, \mu_{y}^{*} \in \mathscr{S}([0,1])$ such that for every $\mu_{x}, \mu_{y} \in \mathscr{S}([0,1])$,

$$
\begin{aligned}
& \int_{0}^{1} \int_{0}^{1} F(x, y) \mu_{x}^{*}(x) \mu_{y}(y) \mathrm{d} y \mathrm{~d} x \\
& \quad \leqslant \int_{0}^{1} \int_{0}^{1} F(x, y) \mu_{x}^{*}(x) \mu_{y}^{*}(y) \mathrm{d} y \mathrm{~d} x \\
& \quad \leqslant \int_{0}^{1} \int_{0}^{1} F(x, y) \mu_{x}(x) \mu_{y}^{*}(y) \mathrm{d} y \mathrm{~d} x .
\end{aligned}
$$

\footnotetext{
${ }^{2}$ This version of the proof was suggested by an anonymous reviewer.
} 
It is evident from (19) that $\left(\mu_{x}^{*}, \mu_{y}^{*}\right)$ defines a saddle point solution in mixed strategies for the game (Başar \& Olsder, 1995, p. 27). In our case $F$ is continuous, hence these results apply. Furthermore, by (19) and the compactness of $[0,1]$,

$$
\begin{aligned}
\int_{0}^{1} & \int_{0}^{1} F(x, y) \mu_{x}^{*}(x) \mu_{y}^{*}(y) \mathrm{d} y \mathrm{~d} x \\
= & \min _{\mu_{x} \in \mathscr{S}([0,1])} \int_{0}^{1} \int_{0}^{1} F(x, y) \mu_{x}(x) \mu_{y}^{*}(y) \mathrm{d} y \mathrm{~d} x \\
& =\min _{x \in[0,1]} \int_{0}^{1} F(x, y) \mu_{y}^{*}(y) \mathrm{d} y .
\end{aligned}
$$

From (18) to (20), we have

$$
\begin{aligned}
& \min _{x \in[0,1]} \int_{0}^{1} F(x, y) \mu_{y}^{*}(y) \mathrm{d} y \\
& =\max _{\mu_{y} \in \mathscr{S}([0,1])} \min _{x \in[0,1]} \int_{0}^{1} F(x, y) \mu_{y}(y) \mathrm{d} y .
\end{aligned}
$$

If we take $\widetilde{\phi}_{u}^{*}=\mu_{y}^{*}$, we then have an optimal solution to (16). This proves the existence of an optimal input.

Theorem 4. For the problem stated in (13) or (16), there is a unique optimal input. Moreover, 0 and 1 do not belong to the support of $\widetilde{\phi}_{u}^{*}$ (or, equivalently, $\underline{\theta}, \bar{\theta} \notin \operatorname{supp} \phi_{u}^{*}$ ), and $\widetilde{\phi}_{u}^{*}$ is symmetric with respect to $\frac{1}{2}$, that is, $\widetilde{\phi}_{u}^{*}(y)=\widetilde{\phi}_{u}^{*}(1-y)$ (i.e. $\left.\phi_{u}^{*}(\omega)=\phi_{u}^{*}(\bar{\theta} \underline{\theta} / \omega)\right)$.

Proof. To prove this, we utilise some results from Karlin (1957). We first need to establish that $f$ is a proper Pólya frequency function. This means, in particular, that we need to show that for every $n \in \mathbb{N}$ and every set of values $\left\{x_{i}\right\}_{i=1, \ldots, n}$ and $\left\{y_{j}\right\}_{j=1, \ldots, n}$ such that $x_{1}<\cdots<x_{n}$ and $y_{1}<\cdots<y_{n}$, the determinant of the matrix $\left(f\left(x_{i}-y_{j}\right)\right)_{i, j}$ is positive. Now,

$f(x-y)=\frac{\mathrm{e}^{k(x-y)}}{\left(\mathrm{e}^{k(x-y)}+1\right)^{2}}=\frac{\mathrm{e}^{k(x+y)}}{\left(\mathrm{e}^{k x}+\mathrm{e}^{k y}\right)^{2}}$.

Then, if we let $z_{i} \triangleq \mathrm{e}^{k x_{i}}>0$ and $w_{j} \triangleq \mathrm{e}^{k y_{j}}>0$, we obtain

$$
\begin{aligned}
\operatorname{sgn} \operatorname{det}\left(f\left(x_{i}-y_{j}\right)\right)_{i, j} & =\operatorname{sgn} \operatorname{det}\left(\frac{w_{i} z_{j}}{\left(w_{i}+z_{j}\right)^{2}}\right)_{i, j} \\
& =\operatorname{sgn} \operatorname{det}\left(\frac{1}{\left(w_{i}+z_{j}\right)^{2}}\right)_{i, j} .
\end{aligned}
$$

The determinant in the last line is given by the following expression, known as Borchardt's identity (Krattenthaler, 1998, p. 29):

$$
\begin{aligned}
& \operatorname{det}\left(\frac{1}{\left(w_{i}+z_{j}\right)^{2}}\right)_{i, j} \\
& =\frac{\prod_{1 \leqslant i<j \leqslant n}\left(w_{j}-w_{i}\right)\left(z_{j}-z_{i}\right)}{\prod_{1 \leqslant i, j \leqslant n}\left(w_{i}+z_{j}\right)} \operatorname{perm}\left(\frac{1}{w_{i}+z_{j}}\right)_{i, j},
\end{aligned}
$$

where perm $X$ is the permanent (Horn \& Johnson, 1985, p. 8) of a square matrix $X$, and it is defined as

$\operatorname{perm}\left(\frac{1}{w_{i}+z_{j}}\right)_{i, j} \triangleq \sum_{\sigma \in S_{n}} \prod_{i=1}^{n} \frac{1}{w_{i}+z_{\sigma(i)}}>0$,

where $S_{n}$ denotes the symmetric group of order $n$ (i.e. the set of all permutations on $\{1, \ldots, n\})$.

From (21), (22) and the ordering of $\left\{x_{i}\right\}_{i=1}^{n}$ and $\left\{y_{j}\right\}_{j=1}^{n}$, we can see that the determinant of $\left(f\left(x_{i}-y_{j}\right)\right)_{i, j}$ is indeed positive.

Now, since $f$ is even, positive, analytic, and a proper Pólya frequency function such that $f^{\prime}(0)=0$, we have by Theorems 1 and 2 of Karlin (1957) that $\widetilde{\phi}_{u}^{*}$ is unique, $0,1 \notin \operatorname{supp} \widetilde{\phi}_{u}^{*}$ and $\widetilde{\phi}_{u}^{*}$ is symmetric with respect to $\frac{1}{2}$.

Theorem 5. For the problem stated in (13) or (16), the optimal input $\left(\phi_{u}^{*}\right.$ or $\widetilde{\phi}_{u}^{*}$, respectively) has finite support. That is, if $\widetilde{\phi}_{u}^{*}$ is such that

$$
\begin{aligned}
& \min _{x \in[0,1]} \int_{0}^{1} f(x-y) \widetilde{\phi}_{u}^{*}(y) \mathrm{d} y \\
& =\max _{\widetilde{\phi}_{u} \in \mathscr{S}([0,1])}\left[\min _{x \in[0,1]} \int_{0}^{1} f(x-y) \widetilde{\phi}_{u}(y) \mathrm{d} y\right]
\end{aligned}
$$

then supp $\widetilde{\phi}_{u}^{*}$ is finite.

Proof. This proof is based on a result in Karlin (1957), which is included here for the sake of completeness. We will focus on the problem stated in (16).

We will first show that if $\mu_{x}^{*}$ is defined as in the proof of Theorem 3 , and $y_{0} \in[0,1]$ is in the support of $\widetilde{\phi}_{u}^{*}$, then

$\int_{0}^{1} f\left(x-y_{0}\right) \mu_{x}^{*}(x) \mathrm{d} x=V_{m}$.

From (19), we have that

$\int_{0}^{1} f(x-y) \mu_{x}^{*}(x) \mathrm{d} x \leqslant V_{m}, \quad y \in[0,1]$.

If this inequality were strict for $y=y_{0}$, then by the continuity of $f$ there would be an interval $[a, b] \subset[0,1]$ for which $a \leqslant y_{0} \leqslant b$ and

$\int_{0}^{1} f(x-y) \mu_{x}^{*}(x) \mathrm{d} x<V_{m}, \quad y \in[a, b]$.

Thus, integrating both sides of (24) weighted by $\widetilde{\phi}_{u}^{*}$, and taking (25) into account, we obtain

$\int_{0}^{1} \int_{0}^{1} f(x-y) \mu_{x}^{*}(x) \widetilde{\phi}_{u}^{*}(y) \mathrm{d} y \mathrm{~d} x<V_{m}$

which contradicts the definition of $V_{m}$. This proves (23).

Now, if supp $\widetilde{\phi}_{u}^{*}$ is infinite, then (23) holds for an infinite number of points in a compact interval, so those points have at least one limit point. On the other hand, the integral of the left side of this expression is an analytic function of $y$ in some region $\Omega$ containing $\mathbb{R}$, and its right side is constant. 
Thus, we have two analytic functions which are equal in a set which has a limit point in $\Omega$, so by a well-known result of complex analysis (Rudin, 1987, p. 209) they must be equal in $\Omega$. In particular it holds that

$\int_{0}^{1} f(x-y) \mu_{x}^{*}(x) \mathrm{d} x=V_{m}, \quad y \in \mathbb{R}$.

However, since $f$ is bounded and $f(u) \rightarrow 0$ for $|u| \rightarrow \infty$,

$\lim _{y \rightarrow \infty} \int_{0}^{1} f(x-y) \mu_{x}^{*}(x) \mathrm{d} x=0 \neq V_{m}$

which contradicts (26). Thus, $\widetilde{\phi}_{u}^{*}$ has finite support.

Remark 6. Theorem 5 basically says that the robust optimal input is a finite linear combination of sinusoids. This is a rather surprising result, since the nominal optimal input is a single sinusoid of frequency equal to $\theta$, so one would expect that the robust optimal signal should have a continuous spectrum to account for all the possible values of this parameter. On the other hand, this property also says that it is very easy to implement such a signal; the only remaining problem is to determine the amplitudes and frequencies of its sinusoids. This is addressed in the Appendix (analytically) and Section 3.4 (numerically).

\subsection{Bandlimited ' $1 / f$ ' noise input}

The results presented above are concerned with the optimal solution to the problem. We will also explore sub-optimal solutions. In the latter context, the following result will be useful.

Lemma 7. Let $\bar{\phi}_{u} \in \mathscr{S}([0,1])$. Also let

$\alpha_{\min }\left(\bar{\phi}_{u}\right) \triangleq \min _{\theta \in \Theta} J\left(\bar{M}\left(\theta, \bar{\phi}_{u}\right), \theta\right)$

$\alpha_{\max }\left(\bar{\phi}_{u}\right) \triangleq \max _{\theta \in \Theta} J\left(\bar{M}\left(\theta, \bar{\phi}_{u}\right), \theta\right)$

Then

$\alpha_{\min }\left(\bar{\phi}_{u}\right) \leqslant \min _{\phi_{u} \in \mathscr{S}\left(\mathbb{R}_{0}^{+}\right)} \max _{\theta \in \Theta} J\left(\bar{M}\left(\theta, \phi_{u}\right), \theta\right) \leqslant \alpha_{\max }\left(\bar{\phi}_{u}\right)$.

Proof. The second inequality follows from the definition of the optimisation problem.

To establish the first inequality, we notice from (27) that

$$
\begin{aligned}
\frac{1}{\alpha_{\min }\left(\bar{\phi}_{u}\right)} & =\frac{1}{\min _{\theta \in \Theta} J\left(\bar{M}\left(\theta, \bar{\phi}_{u}\right), \theta\right)} \\
& =\max _{\theta \in \Theta}\left[J\left(\bar{M}\left(\theta, \bar{\phi}_{u}\right), \theta\right)\right]^{-1} \\
& =\max _{\theta \in \Theta} \int_{\underline{\theta}}^{\bar{\theta}} \frac{\omega^{2} / \theta^{2}}{\left(\omega^{2} / \theta^{2}+1\right)^{2}} \bar{\phi}_{u}(\omega) \mathrm{d} \omega .
\end{aligned}
$$

Thus, for any feasible function $\phi_{u}$, we must have

$\frac{1}{\alpha_{\min }\left(\bar{\phi}_{u}\right)} \geqslant \int_{\underline{\theta}}^{\bar{\theta}} \int_{\underline{\theta}}^{\bar{\theta}} \phi_{u}(\theta) \frac{\omega^{2} / \theta^{2}}{\left(\omega^{2} / \theta^{2}+1\right)^{2}} \bar{\phi}_{u}(\omega) \mathrm{d} \omega \mathrm{d} \theta$.

Now let us assume that the first inequality in (29) is false; i.e.

$$
\begin{aligned}
\alpha_{\min }\left(\bar{\phi}_{u}\right)> & \min _{\phi_{u} \in \mathscr{S}\left(\mathbb{R}_{0}^{+}\right)} \max _{\theta \in \Theta} J\left(\bar{M}\left(\theta, \phi_{u}\right), \theta\right) \\
& =\max _{\theta \in \Theta} J\left(\bar{M}\left(\theta, \phi_{u}^{*}\right), \theta\right)
\end{aligned}
$$

and therefore,

$$
\begin{aligned}
\frac{1}{\alpha_{\min }\left(\bar{\phi}_{u}\right)} & <\min _{\theta \in \Theta}\left[J\left(\bar{M}\left(\theta, \phi_{u}^{*}\right), \theta\right)\right]^{-1} \\
& =\min _{\theta \in \Theta} \int_{\underline{\theta}}^{\bar{\theta}} \frac{\omega^{2} / \theta^{2}}{\left(\omega^{2} / \theta^{2}+1\right)^{2}} \phi_{u}^{*}(\omega) \mathrm{d} \omega .
\end{aligned}
$$

Hence, if we form a convex combination of the integrals on the right-hand side of (31) using $\bar{\phi}_{u}(\theta)$, we must have

$\frac{1}{\alpha_{\min }\left(\bar{\phi}_{u}\right)}<\int_{\underline{\theta}}^{\bar{\theta}} \bar{\phi}_{u}(\theta) \int_{\underline{\theta}}^{\bar{\theta}} \frac{\omega^{2} / \theta^{2}}{\left(\omega^{2} / \theta^{2}+1\right)^{2}} \phi_{u}^{*}(\omega) \mathrm{d} \omega \mathrm{d} \theta$.

However,

$\frac{\omega^{2} / \theta^{2}}{\left(\omega^{2} / \theta^{2}+1\right)^{2}}=\frac{\theta^{2} / \omega^{2}}{\left(\theta^{2} / \omega^{2}+1\right)^{2}}$.

Thus, changing the order of the variables of integration in (32) and using (33) gives

$\frac{1}{\alpha_{\min }\left(\bar{\phi}_{u}\right)}<\int_{\underline{\theta}}^{\bar{\theta}} \int_{\underline{\theta}}^{\bar{\theta}} \phi_{u}^{*}(\theta) \frac{\omega^{2} / \theta^{2}}{\left(\omega^{2} / \theta^{2}+1\right)^{2}} \bar{\phi}_{u}(\omega) \mathrm{d} \theta \mathrm{d} \omega$.

We see that (34) contradicts (30) if we choose $\phi_{u}$ in (30) as $\phi_{u}^{*}$. This contradiction establishes the result.

Remark 8. It is impossible to find an input $\phi_{u}$ which brings $\alpha_{\min }\left(\phi_{u}\right)$ equal to $\alpha_{\max }\left(\phi_{u}\right)$. This is due to the fact that, for a fixed $\phi_{u}$, the cost function $J\left(\bar{M}\left(\theta, \phi_{u}\right), \theta\right)$ is an analytic function of $\theta$ on $\mathbb{R}$, and it vanishes as $|\theta| \rightarrow \infty$; thus, if we force $\alpha_{\min }\left(\phi_{u}\right)=\alpha_{\max }\left(\phi_{u}\right)$, then this cost function would be constant in the interval $[\underline{\theta}, \bar{\theta}]$, which implies, by its analyticity, that it would be constant in $\mathbb{R}$, and hence equal to 0 . This is impossible, since the integral of $\phi_{u}$ over $[\underline{\theta}, \bar{\theta}]$ is equal to 1 , and $\phi_{u}$ can only take nonnegative values over that interval.

Remark 9. We see from Lemma 7 that, if a feasible design, $\phi_{u}$, is found such that $\alpha_{\min }\left(\phi_{u}\right)$ and $\alpha_{\max }\left(\phi_{u}\right)$ are "close", then the corresponding cost function will be "close" to optimal. In particular, if one could choose an input, $\phi_{u}$, such that $\alpha_{\min }\left(\phi_{u}\right)=\alpha_{\max }\left(\phi_{u}\right)$, then this input would have been optimal. Alas, by Remark 8, there is no feasible input which brings $\alpha_{\min }\left(\phi_{u}\right)$ to $\alpha_{\max }\left(\phi_{u}\right)$. However, we will now examine a particular sub-optimal input such that $\alpha_{\min }\left(\phi_{u}\right)$ and $\alpha_{\max }\left(\phi_{u}\right)$ are within a factor of 2 of each other. 
With the above as background, we next consider the following feasible input

$\phi_{u}^{1 / f}(\omega) \triangleq \begin{cases}\frac{1 / \omega}{\ln \bar{\theta}-\ln \underline{\theta}}, & \omega \in[\underline{\theta}, \bar{\theta}], \\ 0 & \text { otherwise. }\end{cases}$

For this input, we have the following result.

Theorem 10. Consider the bandlimited ' $1 / f$ ' noise input given in (35). Let $\alpha_{\min }\left(\phi_{u}^{1 / f}\right)$ and $\alpha_{\max }\left(\phi_{u}^{1 / f}\right)$ be the corresponding limits as in (27) and (28). Then

$$
\begin{aligned}
& \alpha_{\min }\left(\phi_{u}^{1 / f}\right)=2 \ln \left(\frac{\bar{\theta}}{\underline{\theta}}\right) \frac{\bar{\theta}+\underline{\theta}}{\bar{\theta}-\underline{\theta}}, \\
& \alpha_{\max }\left(\phi_{u}^{1 / f}\right)=4 \ln \left(\frac{\bar{\theta}}{\underline{\theta}}\right) \frac{\bar{\theta}^{2}+\underline{\theta}^{2}}{\bar{\theta}^{2}-\underline{\theta}^{2}} .
\end{aligned}
$$

Proof. Upon substitution of (35) on (14), we obtain

$$
\begin{aligned}
\frac{1}{J\left(\bar{M}\left(\theta, \phi_{u}^{1 / f}\right), \theta\right)} & =\frac{1}{\ln \left(\frac{\bar{\theta}}{\underline{\theta}}\right)} \int_{\underline{\theta}}^{\bar{\theta}} \frac{\omega^{2} / \theta^{2}}{\left(\omega^{2} / \theta^{2}+1\right)^{2}} \frac{\mathrm{d} \omega}{\omega} \\
& =\frac{\bar{\theta}^{2}-\underline{\theta}^{2}}{2 \ln \left(\frac{\bar{\theta}}{\underline{\theta}}\right)} \frac{\theta^{2}}{\left(\theta^{2}+\bar{\theta}^{2}\right)\left(\theta^{2}+\underline{\theta}^{2}\right)} .
\end{aligned}
$$

The function $f(\theta)=\theta^{2} /\left[\left(\theta^{2}+\bar{\theta}^{2}\right)\left(\theta^{2}+\underline{\theta}^{2}\right)\right]$, in $\Theta$, increases to a maximum at $\theta=\sqrt{\underline{\theta} \bar{\theta}}$ and then decreases. Finally, since $f(\underline{\theta})=f(\bar{\theta})$ we conclude that

$$
\begin{aligned}
\frac{1}{\alpha_{\max }\left(\phi_{u}^{1 / f}\right)} & =\left.\frac{\bar{\theta}^{2}-\underline{\theta}^{2}}{2 \ln \left(\frac{\bar{\theta}}{\underline{\theta}}\right)} \frac{\theta^{2}}{\left(\theta^{2}+\bar{\theta}^{2}\right)\left(\theta^{2}+\underline{\theta}^{2}\right)}\right|_{\theta=\underline{\theta}} \\
& =\frac{\bar{\theta}^{2}-\underline{\theta}^{2}}{4 \ln \left(\frac{\bar{\theta}}{\underline{\theta}}\right)} \frac{1}{\bar{\theta}^{2}+\underline{\theta}^{2}}
\end{aligned}
$$

and

$$
\begin{aligned}
\frac{1}{\alpha_{\min }\left(\phi_{u}^{1 / f}\right)} & =\left.\frac{\bar{\theta}^{2}-\underline{\theta}^{2}}{2 \ln \left(\frac{\bar{\theta}}{\underline{\theta}}\right)} \frac{\theta^{2}}{\left(\theta^{2}+\bar{\theta}^{2}\right)\left(\theta^{2}+\underline{\theta}^{2}\right)}\right|_{\theta=\sqrt{\underline{\theta} \bar{\theta}}} \\
& =\frac{1}{2 \ln \left(\frac{\bar{\theta}}{\underline{\theta}}\right)} \frac{\bar{\theta}-\underline{\theta}}{\bar{\theta}+\underline{\theta}}
\end{aligned}
$$

which completes the proof.

Corollary 11. For bandlimited ' $1 / f$ ' noise input, the optimal cost, $J^{*}$, must satisfy

$$
\alpha_{\min }\left(\phi_{u}^{1 / f}\right) \leqslant J^{*} \leqslant 2 \alpha_{\min }\left(\phi_{u}^{1 / f}\right) .
$$

Proof. From Theorem 10,

$$
\begin{aligned}
\alpha_{\max }\left(\phi_{u}^{1 / f}\right) & =4 \ln \left(\frac{\bar{\theta}}{\underline{\theta}}\right) \frac{\bar{\theta}^{2}+\underline{\theta}^{2}}{\bar{\theta}^{2}-\underline{\theta}^{2}} \\
& \leqslant 4 \ln \left(\frac{\bar{\theta}}{\underline{\theta}}\right) \frac{\bar{\theta}^{2}+\underline{\theta}^{2}+2 \bar{\theta} \underline{\theta}}{\bar{\theta}^{2}-\underline{\theta}^{2}} \\
& =4 \ln \left(\frac{\bar{\theta}}{\underline{\theta}}\right) \frac{\bar{\theta}+\underline{\theta}}{\bar{\theta}-\underline{\theta}} \\
& =2 \alpha_{\min }\left(\phi_{u}^{1 / f}\right) .
\end{aligned}
$$

The result then follows from Lemma 7.

Remark 12. The above result is rather surprising since it shows that ' $1 / f$ ' noise performs very well for this problem. This is an interesting result since "conventional wisdom" suggests an input more akin to bandlimited white noise (e.g. a PRBS signal). However, one can easily verify that using $\theta=0.1$ and $\bar{\theta}=$ 10 , bandlimited ' $1 / f$ ' noise is almost an order of magnitude superior to bandlimited white noise-see Table 1 presented below.

\subsection{Discrete approximation to the optimal input}

As we have seen in Section 3.2, and as it is well known in the statistics literature (see e.g. Walter \& Pronzato, 1997, p. 340), finding an exact solution to problems of the type (13), (14) is, in general, extremely difficult. Some algorithms have been proposed, e.g. the relaxation algorithm of Shimizu and Aiyoshi (1980). Here we pursue an alternative idea of finding an approximate design by discretisation of the design space (see also Walter \& Pronzato, 1997, p. 341). Since $f$ is continuous, it is well known (Owen, 1968, p. 78) that this approach can approximate the optimal solution as closely as desired.

To develop this approach, we first approximate the integral in Eq. (14) by a Riemann sum. Specifically, utilising Lemma 2 , we choose a grid of $N$ points $\underline{\theta} \leqslant \omega_{m}=\theta_{m} \leqslant \bar{\theta}$ for $0 \leqslant m \leqslant N$ such that $\omega_{0}=\theta_{0}=\underline{\theta}, \omega_{N}=\theta_{N}=\bar{\theta}$. Then

$$
\begin{aligned}
J_{m} & \triangleq\left[\int_{\underline{\theta}}^{\bar{\theta}} \frac{\omega^{2} / \theta_{m}^{2}}{\left(\omega^{2} / \theta_{m}^{2}+1\right)^{2}} \phi_{u}(\omega) \mathrm{d} \omega\right]^{-1} \\
& \approx\left[\sum_{n=0}^{N-1} \frac{\omega_{n}^{2} / \theta_{m}^{2}}{\left(\omega_{n}^{2} / \theta_{m}^{2}+1\right)^{2}} \phi_{u}\left(\omega_{n}\right)\left(\omega_{n+1}-\omega_{n}\right)\right]^{-1} \\
& =\left[\sum_{n=0}^{N-1} A_{m, n} E_{n}\right]^{-1}
\end{aligned}
$$

where $A_{m, n} \triangleq\left(\omega_{n}^{2} / \theta_{m}^{2}\right) /\left[\left(\omega_{n}^{2} / \theta_{m}^{2}+1\right)^{2}\right]>0$ and $E_{n} \triangleq \phi_{u}\left(\omega_{n}\right)$ $\left(\omega_{n+1}-\omega_{n}\right)$. Notice that the matrix $A=\left\{A_{m, n}\right\}$ is symmetric and has positive entries. 
Table 1

Relative values of cost for the different input signals

\begin{tabular}{|c|c|c|c|}
\hline & $\max _{\theta \in \Theta}\left[\theta^{2} \bar{M}\left(\theta, \phi_{u}\right)\right]^{-1}$ & Bayesian cost on $\theta$ & Bayesian cost on $\ln \theta$ \\
\hline Single frequency at $\omega=1$ & 7.75 & 4.8 & 2.26 \\
\hline Bandlimited white noise & 12.09 & 9.05 & 2.96 \\
\hline Bandlimited ' $1 / f$ ' noise & 1.43 & 1.51 & 1.07 \\
\hline Robust min-max optimal input & 1.00 & 1.45 & 1.12 \\
\hline Bayesian design (for uniform distribution on $\theta$ ) & 5.4 & 1.00 & 1.61 \\
\hline Bayesian design (for uniform distribution on $\ln \theta$ ) & 1.53 & 1.46 & 1.00 \\
\hline
\end{tabular}

We can now state the following discrete approximation to the optimisation problem in Eq. (13):

$\mathbf{E}^{*}=\arg \min _{\mathbf{E} \in \mathscr{S}_{d}} \max _{0 \leqslant m<N}\left(\mathbf{e}_{m}^{\mathrm{T}} A \mathbf{E}\right)^{-1}$,

where $\mathscr{S}_{d} \triangleq\left\{\mathbf{E} \in \mathbb{R}^{N}: \mathbf{1}^{\mathrm{T}} \mathbf{E}=1, E_{n} \geqslant 0\right\}, \mathbf{E} \triangleq\left[\begin{array}{lll}E_{0} & \cdots & E_{N-1}\end{array}\right]^{\mathrm{T}}$, $\mathbf{e}_{m}$ is the $m$ th column of the $N$-dimensional identity matrix, and $\mathbf{1}$ is an $\mathrm{N}$-dimensional vector of ones.

It is well known that a finite dimensional min-max optimisation problem, such as (37), can be converted into a standard linear programming (LP) problem; see McKinsey (1952, p. 296), Dantzig (1951), and Gale, Kuhn, and Tucker (1951).

It is also quite straightforward to compute a discrete approximation to the Bayesian optimal input for the example problem. For example, say that we use $J\left(\bar{M}\left(\theta, \phi_{u}\right), \theta\right)$ as a Bayesian risk and, for the sake of illustration, assume that $\theta$ has a uniform distribution on $\Theta$. Then, the Bayesian design problem becomes

$$
\begin{aligned}
\phi_{u}^{B}= & \arg \min _{\phi_{u} \in \mathscr{S}\left(\mathbb{R}_{0}^{+}\right)} \frac{1}{\bar{\theta}-\underline{\theta}} \\
& \times \int_{\underline{\theta}}^{\bar{\theta}}\left[\int_{0}^{\infty} \frac{\omega^{2} / \theta^{2}}{\left(\omega^{2} / \theta^{2}+1\right)^{2}} \phi_{u}(\omega) \mathrm{d} \omega\right]^{-1} \mathrm{~d} \theta .
\end{aligned}
$$

We can approximate this, as in (36), by

$$
\begin{aligned}
\mathbf{E}^{B}= & \arg \min _{\mathbf{E} \in \mathscr{S}_{d}} \frac{1}{N} \sum_{k=0}^{N-1} \exp \left\{\left[\frac{\ln \bar{\theta}-\ln \underline{\theta}}{N}\right] k+\ln \underline{\theta}\right\} \\
& \times\left(\mathbf{e}_{k}^{\mathrm{T}} A \mathbf{E}\right)^{-1} .
\end{aligned}
$$

In the next section, we will also consider a Bayesian design for the case when $\ln \theta$ has a uniform distribution on $(\ln \underline{\theta}, \ln \bar{\theta})$.

\subsection{Numerical results}

We present below numerical results for the problem described above where we take $\underline{\theta}=0.1, \bar{\theta}=10, N=100$ and compare:

(i) A nominal input of frequency $1 \mathrm{rad} / \mathrm{s}$ (notice that this is the optimal input if the initial estimate of the parameter is $\widehat{\theta}=1)$.

(ii) Bandlimited white noise input, limited to the frequency range $[0.1,10] \mathrm{rad} / \mathrm{s}$.

(iii) Bandlimited ' $1 / f$ ' noise input, limited to the frequency range $[0.1,10] \mathrm{rad} / \mathrm{s}$.

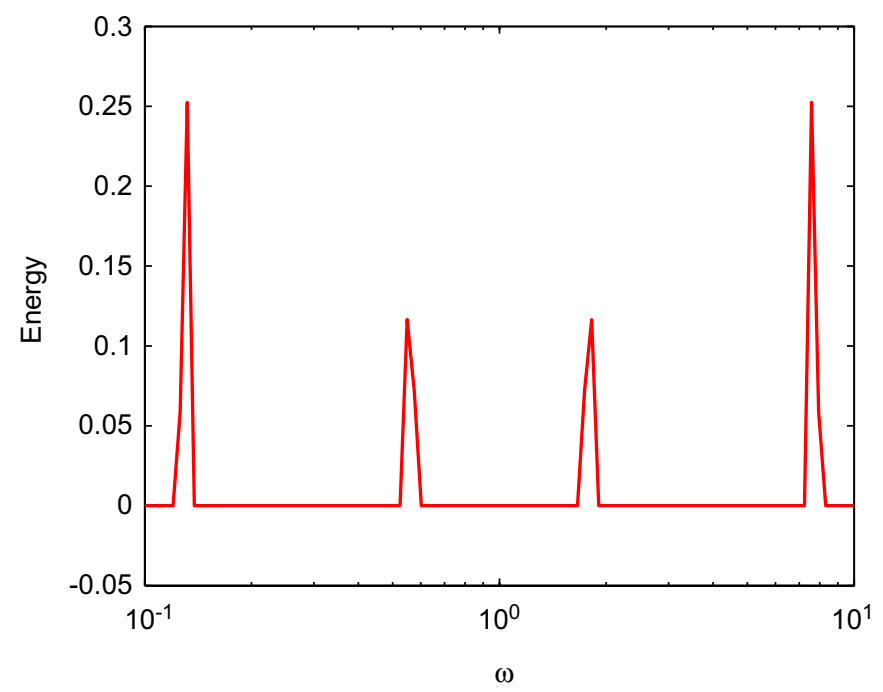

Fig. 2. Values of $E$ for the discretised robust optimal input.

(iv) The approximate discretised robust optimal input generated by LP.

(v) The approximate discretised Bayesian optimal input for a uniform distribution on $\theta$.

(vi) The approximate discretised Bayesian optimal input for a uniform distribution on $\ln \theta$.

Relative costs for the different experimental conditions are shown in Table 1. Notice that the costs have been scaled so that the optimal value is 1 .

We see from Table 1 that bandlimited white noise gives poor performance under all criteria. Indeed, we see from the table that bandlimited ' $1 / f$ ' noise is almost an order of magnitude better than a bandlimited white noise input for all cost functions. Furthermore, the discretised min-max optimum gives a further $40 \%$ improvement for the min-max cost function. The discretised min-max optimal input energy, $\phi_{u}^{*}$, is shown in Fig. 2. Notice that the above results are consistent with Theorem 5 , which asserts that the unique optimal input has finite support. The corresponding values of $\left[\theta^{2} \bar{M}\left(\theta, \phi_{u}^{*}\right)\right]^{-1}$ as a function of $\theta$ are shown in Fig. 1, where they can be compared to the corresponding values for the nominal optimal input and bandlimited ' $1 / f$ ' noise. It is interesting to notice from Fig. 1 that $\left[\theta^{2} \bar{M}\left(\theta, \phi_{u}^{*}\right)\right]^{-1}$ is an almost constant function of $\theta$. This should be compared with the comments in Remark 9. The comparative costs are given in Table 1. 


\section{Generalisation to multi-parameter problems}

For the multi-parameter case we return to the general expression for $\bar{M}(\beta, \phi)$ given in (5) and (6). Again for simplicity, we assume white noise $\left(G_{2}(q)=1\right)$ and hence refer only to $\theta$ although the extension to coloured noise offers no additional difficulties. We convert this problem into an approximate discrete form as was done in Section 3 by discretising the input and parameter spaces. We write

$$
Q_{k}(\mathbf{E}) \triangleq \sum_{m} A_{k m} E_{m}, \quad \theta_{k} \in \Theta
$$

as an approximation to the integral in (6) i.e. $Q_{k}$ is the information matrix corresponding to the $k$ th (discretised) element $\theta_{k}$ of the parameter set $\Theta$, the index $m$ denotes the frequency and $E_{m}$ is the input energy at the $m$ th frequency.

There exist many possible choices for the inner design criterion $J\left(\bar{M}\left(\theta, \phi_{u}\right), \theta\right)$ in the multi-parameter case-see the discussion in Section 2.2 and recent results in Welsh, Goodwin, and Feuer (2006). Three alternatives are discussed below.

\subsection{Minimal eigenvalue}

The use of the minimum eigenvalue of the information matrix as a design criterion for nominal experiment design has previously been studied (Mareels et al., 1987). For the robust case, we propose to optimise the worst case of the following related criterion which uses the minimum eigenvalue of a scaled version of the information matrix

$J_{1}\left(\bar{M}\left(\theta, \phi_{u}\right), \theta\right) \triangleq\left(\lambda_{\min }\left\{S_{\theta} \bar{M}\left(\theta, \phi_{u}\right) S_{\theta}\right\}\right)^{-1}$,

where $\lambda_{\text {min }}$ denotes the minimum eigenvalue and $S_{\theta}$ is a parameter dependent scaling matrix. One possible choice for $S_{\theta}$ is $\operatorname{diag}\left[\theta_{1}, \ldots, \theta_{m}\right]$. The motivation for this choice is that $\bar{M}\left(\theta, \phi_{u}\right)^{-1}$ is a measure of the parameter covariance matrix. Hence $S_{\theta}^{-1} \bar{M}\left(\theta, \phi_{u}\right)^{-1} S_{\theta}^{-1}$ is the covariance normalised by the nominal values of each parameter. Therefore it is a measure of the relative error. This seems to be an important property in the robust design context (where we maximise over $\theta \in \Theta$ ) since it ensures that one is maximising (over $\Theta$ ) the relative errors. These errors are normalised and thus better scaled for comparison purposes.

Another useful property of $J_{1}\left(\bar{M}\left(\theta, \phi_{u}\right), \theta\right)$ is that, due to the normalisation by $S_{\theta}$, the scaled information matrix does not depend on the system gain. This simplifies the problem of discretisation of the set $\Theta$ by eliminating one degree of freedom (the gain). This also makes sense, heuristically speaking, since the system gain simply scales the output.

\subsection{Relative frequency domain errors}

This criterion is motivated by robust control (Zhou et al., 1996). It is well known (Goodwin, Graebe, \& Salgado, 2001, p. 147), that the achieved sensitivity, $S$, is related to the nominal sensitivity, $S_{0}$, via

$$
S=\frac{S_{0}}{1+T_{0} \Delta G / G}
$$

where $T_{0}$ is the nominal complementary sensitivity and $\Delta G / G$ is the relative error in $G$. Indeed, this leads to the well-known sufficient condition for robust stability, namely $\left\|T_{o} \Delta G / G\right\|_{\infty}<1$.

Say we put an upper bound on $\left\|T_{0}\right\|_{\infty}$, then we see that what is important is the infinity norm of the relative error in $G, \Delta G / G$. Then, noting that the covariance of all unbiased estimates of $\theta$ are lower bounded by $(N \bar{M})^{-1}$ where $N$ is the number of data points (see Goodwin \& Payne, 1977, p. 6), we can obtain a measure of the size of $\Delta G / G$ as

$$
\begin{aligned}
\left\|\frac{N \mathbb{E}|\Delta G|^{2}}{|G|^{2}}\right\|_{\infty} & =\max _{\omega} \frac{\frac{\partial G(\mathrm{j} \omega)^{T}}{\partial \theta} \bar{M}^{-1} \frac{\partial G(-\mathrm{j} \omega)}{\partial \theta}}{|G(\mathrm{j} \omega)|^{2}} \\
& \triangleq J_{2}\left(\bar{M}\left(\theta, \phi_{u}\right), \theta\right) .
\end{aligned}
$$

Note that here we use the per-sample information matrix $\bar{M}$.

It is readily seen that $J_{2}\left(\bar{M}\left(\theta, \phi_{u}\right), \theta\right)$ is a dimensionless quantity. Thus the associated experiment design is independent of the system gain in the same way that this was true for $J_{1}$ (see (38)).

Remark 13. We see that the criterion $J_{2}$ has the form

$J_{2}\left(\bar{M}\left(\theta, \phi_{u}\right), \theta\right)=\max _{\omega} g(\omega)^{H} \bar{M}^{-1} g(\omega)$,

where

$g(\omega) \triangleq \frac{\partial G(-\mathrm{j} \omega) / \partial \theta}{|G(\mathrm{j} \omega)|}$

Thus we see that (39) maximises $x^{H} \bar{M}^{-1} x$ where $x$ is restricted to the particular set of vectors given in (40). This can be compared with $J_{1}\left(\bar{M}\left(\theta, \phi_{u}\right), \theta\right)$ which is actually equivalent to maximising $y^{H} \bar{M}^{-1} y$ over the set of vectors $y$ where $y=S_{\theta} Z$ and $Z^{H} Z=1$.

\subsection{A criterion related to the $v$ gap}

Hildebrand and Gevers (2003a, 2003b) have suggested the following criterion for nominal experiment design such that the worst case $v$ gap is minimised:

$$
\begin{aligned}
& J_{3}\left(\bar{M}\left(\theta, \phi_{u}\right), \theta\right) \\
& \left.\triangleq \max _{\omega} \frac{\lambda_{\max }\left\{[ \begin{array} { c } 
{ \operatorname { R e } \frac { \partial G } { \partial \theta } } \\
{ \operatorname { I m } \frac { \partial G } { \partial \theta } }
\end{array} ] \overline { M } ^ { - 1 } \left[\operatorname{Re} \frac{\partial G}{\partial \theta}\right.\right.}{\left.\left.\operatorname{Im} \frac{\partial G}{\partial \theta}\right]\right\}}\right|_{\omega} \\
& =\max _{\omega} \frac{\lambda_{\max }\left[\begin{array}{cc}
R_{\omega}^{\mathrm{T}} \bar{M}^{-1} R_{\omega} & R_{\omega}^{\mathrm{T}} \bar{M}^{-1} I_{\omega} \\
I_{\omega}^{\mathrm{T}} \bar{M}^{-1} R_{\omega} & I_{\omega}^{\mathrm{T}} \bar{M}^{-1} I_{\omega}
\end{array}\right]}{\left[1+\left|G_{\omega}\right|^{2}\right]^{2}},
\end{aligned}
$$


where the subscript $\omega$ denotes 'frequency $\omega$ ', $R_{\omega} \triangleq \operatorname{Re}\{\partial G(\omega) /$ $\partial \theta\}$ and $I_{\omega} \triangleq \operatorname{Im}\{\partial G(\omega) / \partial \theta\}$.

Remark 14. Not surprisingly, there is a connection between $J_{2}\left(\bar{M}\left(\theta, \phi_{u}\right), \theta\right)$ and $J_{3}\left(\bar{M}\left(\theta, \phi_{u}\right), \theta\right)$ since both are motivated by robust control. Specifically, it is readily seen that

$\frac{\partial G^{T}}{\partial \theta} \bar{M}^{-1} \frac{\partial \bar{G}}{\partial \theta}=R_{\omega}^{\mathrm{T}} \bar{M}^{-1} R_{\omega}+I_{\omega}^{T} \bar{M}^{-1} I_{\omega}=\operatorname{tr} L_{\omega}$,

where $L_{\omega}$ appears in (41), i.e.

$L_{\omega} \triangleq\left[\begin{array}{cc}R_{\omega}^{T} \bar{M}^{-1} R_{\omega} & R_{\omega}^{T} \bar{M}^{-1} I_{\omega} \\ I_{\omega}^{T} \bar{M}^{-1} R_{\omega} & I_{\omega}^{T} \bar{M}^{-1} I_{\omega}\end{array}\right]$.

We notice that $\lambda_{\max }\left(L_{\omega}\right) \leqslant \operatorname{tr} L_{\omega} \leqslant 2 \lambda_{\max }\left(L_{\omega}\right)$. Hence, we see that the criteria $J_{2}\left(\bar{M}\left(\theta, \phi_{u}\right), \theta\right)$ and $J_{3}\left(\bar{M}\left(\theta, \phi_{u}\right), \theta\right)$ are loosely connected. Moreover, Remark 13 links both criteria to $J_{1}\left(\bar{M}\left(\theta, \phi_{u}\right), \theta\right)$.

A potential issue with the criterion $J_{3}\left(\bar{M}\left(\theta, \phi_{u}\right), \theta\right)$ is that, unlike $J_{1}\left(\bar{M}\left(\theta, \phi_{u}\right), \theta\right)$ and $J_{2}\left(\bar{M}\left(\theta, \phi_{u}\right), \theta\right)$, it is not dimensionless. This is not an issue in the case of nominal experiment design. However, it could be a problem with respect to robust design when one wishes to compare the criteria for different values of $\theta \in \Theta$. A possible normalisation for $J_{3}\left(\bar{M}\left(\theta, \phi_{u}\right), \theta\right)$ is given in Welsh et al. (2006).

Remark 15. Notice that the above criteria are convex in terms of $\phi_{u}$. This follows since the supremum of a family of convex functions is itself convex.

\section{Numerical example}

To illustrate the merits of the robust optimal experiment design procedure on a realistic example, we have evaluated a discretised approximation to each of the criteria $J_{1}(\bar{M}, \theta), J_{2}(\bar{M}, \theta)$ and $J_{3}(\bar{M}, \theta)$ on a multi-parameter design example. The system is given by $G_{2}(s)=1$ and

$G_{1}(s)=\frac{K}{s^{2}+a_{1} s+a_{0}}$.

We assume prior knowledge of the parameters as follows:

$\theta_{1} \triangleq a_{1} \in[1,2], \quad \theta_{2} \triangleq a_{0} \in[1,9], \quad \theta_{3} \triangleq K \in[1,2]$.

The parameter and frequency ranges were all divided into logarithmically spaced grids for the optimisation. For our example we chose each range to contain 20 values. We also used the Matlab ${ }^{\circledR}$ optimisation toolbox to carry out the min-max designs.
In all our simulations we approximated the integral in (6) by the following discretisation:

$$
\begin{aligned}
\bar{M}\left(\theta, \phi_{u}\right) & =\int_{0}^{\infty} \operatorname{Re}\{Q(\omega)\} \phi_{u}(\omega) \mathrm{d} \omega \\
& \approx \sum_{n=1}^{20} \operatorname{Re}\left\{Q\left(\omega_{n}\right)\right\} \int_{\Delta_{n}} \phi_{u}(\omega) \mathrm{d} \omega \\
& =\sum_{n=1}^{20} \operatorname{Re}\left\{Q\left(\omega_{n}\right)\right\} E_{n},
\end{aligned}
$$

where

$Q(\omega) \triangleq \frac{\partial G_{1}(\mathrm{j} \omega)}{\partial \theta}\left|G_{2}(\mathrm{j} \omega)\right|^{-2}\left[\frac{\partial G_{1}(\mathrm{j} \omega)}{\partial \theta}\right]^{H}$

and $E_{n} \triangleq \int_{\Delta_{n}} \phi_{u}(\omega) \mathrm{d} \omega$ is the input energy in the frequency range $\Delta_{n}$. We have chosen $\Delta_{n} \triangleq \omega_{n+1}-\omega_{n}$, where $\omega_{n}=0.3(10)^{(n-1) / 20}$

The discrete approximation to the robust optimal input was found for each of the criteria $\max _{\theta} J_{1}, \max _{\theta} J_{2}$ and $\max _{\theta} J_{3}$. For those criteria depending on a maximum over a frequency range (i.e. $\max _{\theta} J_{2}$ and $\max _{\theta} J_{3}$ ), we limited $\omega$ to $[0.3,3] \mathrm{rad} / \mathrm{s}$. (This choice was motivated by the region of possible pole locations.)

Sample results are shown in Fig. 3. Fig. 3(a) shows the discretised optimal input energy distribution for criterion $\max _{\theta} J_{1}$. Notice again that the input has finite support. Fig. 3(b) shows the discretised optimal input energy for $\max _{\theta} J_{2}$. We see from Figs. 3(a) and (b) that the optimal input is roughly the same whether we use $\max _{\theta} J_{1}$ or $\max _{\theta} J_{2}$. Fig. 3(c) shows the discretised optimal input energy distribution for $\max _{\theta} J_{3}$. Finally, Table 2 compares the evaluated cost functions obtained with different design criteria for different inputs. The table also shows the values of the corresponding cost functions for bandlimited ' $1 / f$ ' noise and bandlimited white noise.

Our choice of bandlimited ' $1 / f$ ' noise is motivated by earlier results in Section 3 where we showed that bandlimited ' $1 / f$ ' noise was near optimal for robust experiment design for the illustrative problem.

The results in Table 2 have been normalised so that the optimal design gives a value of 1 . Observations from this table are:

(1) Bandlimited ' $1 / f$ ' noise gives much better results in the multi-parameter case for all criteria than does bandlimited white noise. We believe this to be a surprising and interesting observation!

(2) For $\max _{\theta} J_{1}$, the discretised robust optimal input is approximately twice as good as bandlimited ' $1 / f$ ' noise and about 5 times as good as bandlimited white noise.

(3) For $\max _{\theta} J_{2}$, the discretised optimal input is about 1.7 times better than the optimal input for $\max _{\theta} J_{1}$. The robust optimal input for $\max _{\theta} J_{2}$ is about 3 times as good as bandlimited ' $1 / f$ ' noise and almost 9 times as good as bandlimited white noise. 
a

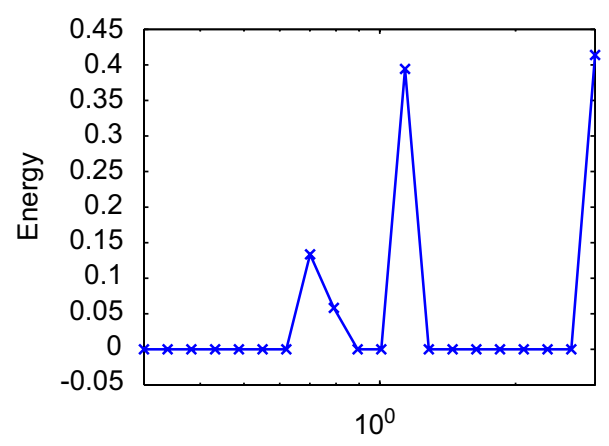

Frequency $(\mathrm{rad} / \mathrm{sec})$ b

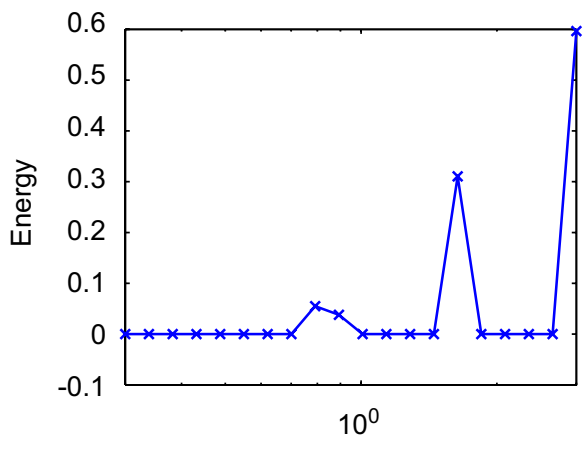

Frequency $(\mathrm{rad} / \mathrm{sec})$

C

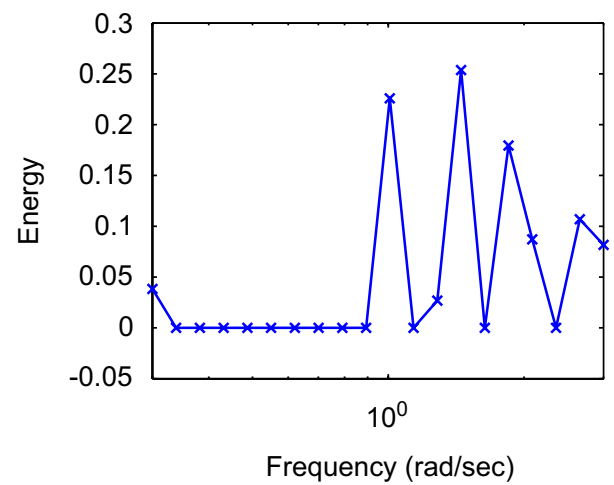

Fig. 3. Values of $E$ for the discretised robust optimal input obtained from $\operatorname{criteria} \max _{\theta} J_{1}$ (a), $\max _{\theta} J_{2}$ (b) and $\max _{\theta} J_{3}$ (c).

Table 2

Values of cost for the criteria

\begin{tabular}{llll}
\hline & $\max _{\theta} J_{1}$ & $\max _{\theta} J_{2}$ & $\max _{\theta} J_{3}$ \\
\hline Optimal input for $\max _{\theta} J_{1}$ & 1 & 1.73 & 1.55 \\
Optimal input for $\max _{\theta} J_{2}$ & 1.14 & 1 & 1.32 \\
Optimal input for $\max _{\theta} J_{3}$ & 1.78 & 2.43 & 1 \\
Bandlimited ' $1 / f$ ' noise & 2.08 & 3.13 & 1.22 \\
Bandlimited white noise & 5 & 8.8 & 1.93 \\
\hline
\end{tabular}

(4) For $\max _{\theta} J_{3}$, the criterion seems to be less sensitive to the test signal.

(5) We also notice that the discretised optimal inputs for $\max _{\theta} J_{1}$ and $\max _{\theta} J_{2}$ are quite similar whilst the discretised optimal result for $\max _{\theta} J_{3}$ is considerably different.

To further motivate our robust design approach, we also tried nominal experiment design for this example. Here we assumed nominal parameter values in the centre of the a priori region, i.e. we chose $\hat{\theta}_{1}=1.5, \hat{\theta}_{2}=5$ and $\hat{\theta}_{3}=1.5$. We then found the corresponding exact nominal optimal input using $J_{1}$ as our design criterion. For this input, in the case where the true parameters take any value in the a priori region, we found that the range of the cost is 30-2700. This lies in stark contrast to the range of cost for the discretised robust optimal input which turns out to be $26-400$. Thus, we see that the discretised robust optimal input gives almost $700 \%$ improvement in the worst case performance relative to the nominal optimal input. However, this is achieved with a negligible change (10\%) in the best case performance, which provides a strong incentive to move to a robust design criterion.

\section{Conclusion}

This paper has described and analysed a min-max approach to robust optimal experiment design for dynamic system identification. The paper has also evaluated and compared several different design criteria. Two illustrative examples have been presented, one with a scalar parameter and the other with multiple parameters, showing that substantial improvements in the worst case performance are achieved using a discretised robust design procedure relative to what is achieved via nominal experiment design procedure. The paper also opens up many potential research issues. For example, it is surprising that bandlimited ' $1 / f$ ' noise performs so well. This suggests that we should not use (near) white inputs such as PRBS. Instead, it may be valuable to investigate binary inputs whose energy distribution approximates bandlimited ' $1 / f$ ' noise. Also, the results in Section 5 suggest that the problem of linking robust control and experiment design may still offer many interesting research challenges. 


\section{Acknowledgements}

The authors acknowledge helpful comments made by Juan Carlos Agüero, Milan Derpich, Michel Gevers, Arkadi Nemirovski and Brett Ninness on earlier drafts of this paper. Also, valuable suggestions were made by two anonymous reviewers. These are gratefully acknowledged.

\section{Appendix A. Explicit solution of the robust experiment de- sign problem}

To obtain an explicit solution to the robust experiment design problem for the illustrative example, we will use some ideas ${ }^{3}$ from Bohnenblust, Karlin, and Shapley (1950), McKinsey (1952, Chapter 12), and Karlin (1957).

From Section 3.2, the problem can be stated as

$$
\begin{aligned}
\mu_{y}^{*}= & \arg \max _{\mu_{y} \in \mathscr{S}([0,1])} \min _{\mu_{x} \in \mathscr{S}([0,1])} \\
& \times \int_{0}^{1}\left[\int_{0}^{1} \frac{\mathrm{e}^{k(x-y)}}{\left(\mathrm{e}^{k(x-y)}+1\right)^{2}} \mu_{x}(x) \mathrm{d} x\right] \mu_{y}(y) \mathrm{d} y .
\end{aligned}
$$

The kernel of the associated game is $F(x, y) \triangleq f(x-y)$, where $f(u) \triangleq \mathrm{e}^{k u} /\left(\mathrm{e}^{k u}+1\right)^{2}$.

Let us denote the optimal mixed strategies of players $x$ and $y$ of game (42) by $\mu_{x}^{*}$ and $\mu_{y}^{*}\left(=\widetilde{\phi}_{u}^{*}\right)$, respectively. Also, let

$$
\begin{aligned}
& \Psi_{x}(x) \triangleq \int_{0}^{1} \frac{\mathrm{e}^{k(x-y)}}{\left(\mathrm{e}^{k(x-y)}+1\right)^{2}} \mu_{y}^{*}(y) \mathrm{d} y, \\
& \Psi_{y}(y) \triangleq \int_{0}^{1} \frac{\mathrm{e}^{k(x-y)}}{\left(\mathrm{e}^{k(x-y)}+1\right)^{2}} \mu_{x}^{*}(x) \mathrm{d} x .
\end{aligned}
$$

When $k$ is very small, $F(x, y)$ is strictly concave in $y \in[0,1]$ for every $x \in[0,1]$. Thus, $\Psi_{y}(y)$ (which appears in (42) by taking $\mu_{x}=\mu_{x}^{*}$ ) is a linear combination of strictly concave functions in $y$, so it is strictly concave as well, and it has a unique maximum at $y=\frac{1}{2}$ (because of the symmetry of $F$ ). This means that

$$
\widetilde{\phi}_{u}^{*}(y)=\mu_{y}^{*}(y)=\delta(y-1 / 2) .
$$

Notice that this coincides with the single sinusoid robust design given in Walter and Pronzato (1997, p. 339). On the other hand, by the Minimax Theorem (Glicksberg, 1950), $\mu_{x}^{*}$ must satisfy

$\mu_{x}^{*}=\arg \min _{\mu_{x} \in \mathscr{S}([0,1])} \int_{0}^{1} \frac{\mathrm{e}^{k(x-1 / 2)}}{\left(\mathrm{e}^{k(x-1 / 2)}+1\right)^{2}} \mu_{x}(x) \mathrm{d} x$,

that is,

$\mu_{x}^{*}(x)=\frac{1}{2} \delta(x)+\frac{1}{2} \delta(x-1)$.

If we increase the value of $k, F(x, y)$ eventually ceases to be strictly concave in $y$ for every $x$. This implies that there is a number $k_{1} \in \mathbb{R}^{+}$such that, for $k>k_{1}, \Psi_{y}(y)$ has at least two maxima. This value can be computed by setting the second

\footnotetext{
3 The idea behind this method was suggested by an anonymous reviewer.
}

derivative of $\Psi_{y}(y)$ equal to zero for $y=\frac{1}{2}$, which gives an equation whose only positive root is $k_{1}=2 \ln (2+\sqrt{3}) \approx 2.6339$.

Thus, (43) and (44) hold for $0<k \leqslant k_{1}$. However, for values of $k$ slightly greater than $k_{1}$, (44) still holds, so $\Psi_{y}(y)$, with $\mu_{x}^{*}$ given by (44), has two maxima at, say, $y^{\prime}$ and $1-y^{\prime}$, where

$$
\left.\frac{\partial}{\partial y}\left[\int_{0}^{1} \frac{\mathrm{e}^{k(x-y)}}{\left(\mathrm{e}^{k(x-y)}+1\right)^{2}} \mu_{x}^{*}(x) \mathrm{d} x\right]\right|_{y=y^{\prime}}=0 .
$$

This equation has only one real solution $y^{\prime}$ between 0 and $\frac{1}{2}$, from which we obtain

$\widetilde{\phi}_{u}^{*}(y)=\mu_{y}^{*}(y)=\frac{1}{2} \delta\left(y-y^{\prime}\right)+\frac{1}{2} \delta\left(y-\left[1-y^{\prime}\right]\right)$.

Expressions (44) and (45) hold as long as $\Psi_{y}(y)$ has two maxima, which is true while (44) satisfies

$$
\begin{array}{r}
\mu_{x}^{*}=\arg \min _{\mu_{x} \in \mathscr{S}([0,1])} \int_{0}^{1} \Psi_{x}(x) \mu_{x}(x) \mathrm{d} x \\
=\arg \min _{\mu_{x} \in \mathscr{S}([0,1])} \int_{0}^{1} \frac{1}{2}\left[\frac{\mathrm{e}^{k\left(x-y^{\prime}\right)}}{\left(\mathrm{e}^{k\left(x-y^{\prime}\right)}+1\right)^{2}}\right. \\
\left.+\frac{\mathrm{e}^{k\left(x-\left(1-y^{\prime}\right)\right)}}{\left(\mathrm{e}^{k\left(x-\left(1-y^{\prime}\right)\right)}+1\right)^{2}}\right] \mu_{x}(x) \mathrm{d} x .
\end{array}
$$

$\Psi_{x}(x)$ has local minima at $x=0,1$ and $\frac{1}{2}$, so (44) and (45) hold for $k_{1}<k \leqslant k_{2}$, where $k_{2} \in \mathbb{R}^{+}$is such that

$$
\begin{gathered}
\left.\frac{\partial}{\partial y}\left[\int_{0}^{1} \frac{\mathrm{e}^{k_{2}(x-y)}}{\left(\mathrm{e}^{k_{2}(x-y)}+1\right)^{2}} \mu_{x}^{*}(x) \mathrm{d} x\right]\right|_{y=y^{\prime}}=0, \\
\frac{\mathrm{e}^{k_{2}\left(1 / 2-y^{\prime}\right)}}{\left(\mathrm{e}^{k_{2}\left(1 / 2-y^{\prime}\right)}+1\right)^{2}}+\frac{\mathrm{e}^{k_{2}\left(y^{\prime}-1 / 2\right)}}{\left(\mathrm{e}^{k_{2}\left(y^{\prime}-1 / 2\right)}+1\right)^{2}} \\
=\frac{\mathrm{e}^{-k_{2} y^{\prime}}}{\left(\mathrm{e}^{-k_{2} y^{\prime}}+1\right)^{2}}+\frac{\mathrm{e}^{-k_{2}\left(1-y^{\prime}\right)}}{\left(\mathrm{e}^{-k_{2}\left(1-y^{\prime}\right)}+1\right)^{2}} .
\end{gathered}
$$

The first equation gives $y^{\prime}$ in terms of $k=k_{2}$, and the last equation gives the minimal value of $k$, say $k_{2}$, for which $x=\frac{1}{2}$ is a global minimum of $\Psi_{x}(x)$. This system of equations gives $k_{2} \approx 3.6855$.

For values of $k$ slightly higher than $k_{2}$, (45) still holds, but (44) changes to

$\mu_{x}^{*}(x)=\alpha \delta(x)+(1-2 \alpha) \delta(x-1 / 2)+\alpha \delta(x-1)$,

where $\alpha \in[0,0.5]$. This expression satisfies (46) for every $\alpha$, but we must assure that (45) still satisfies (42) when using (47), which happens for a particular choice of $\alpha$. One way to find the optimal value of $\alpha$ is to substitute (47) into (42) and to force the derivative of the integral in (42) with respect to $y$ equal to zero for $y=y^{\prime}$. (See the proof of Theorem 12.5 of McKinsey, 1952 for an example of how to use this idea in convex games.)

Continuing in this way, we can see that it is possible, at least in principle, to get an "explicit" solution to the robust experiment design problem. 

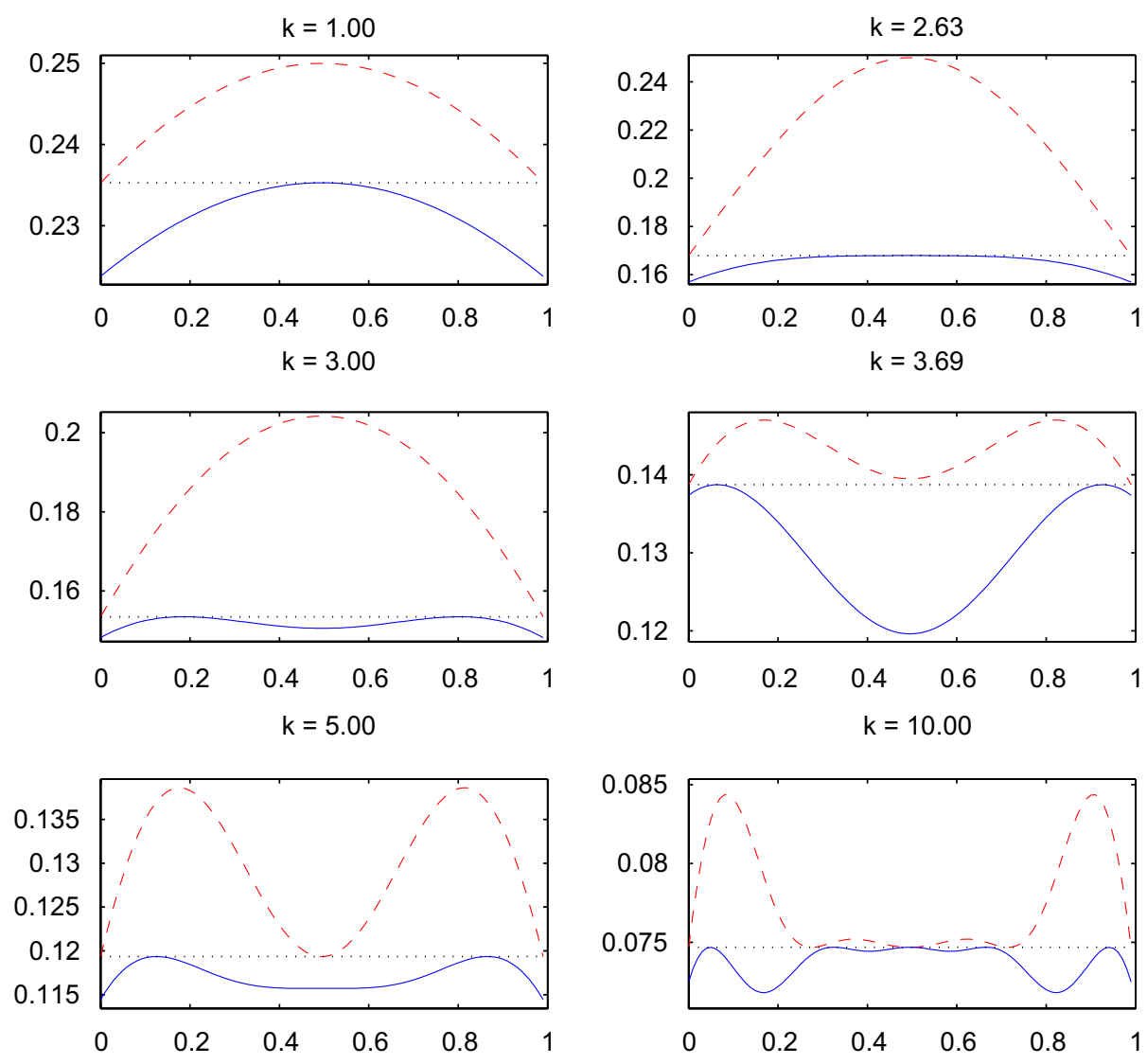

Fig. 4. $\Psi_{x}$ (dashed) and $\Psi_{y}$ (solid) for various values of $k$. The dotted line corresponds to the average value, $V_{m}$.

In Fig. 4 we can see the shapes of $\Psi_{x}$ and $\Psi_{y}$ for various values of $k$. These figures have been generated by an LP algorithm as explained in Section 3.4. We can also see from the figures that the minimum value of $\Psi_{x}$ coincides with the maximum value of $\Psi_{y}$; this is a consequence of the Minimax Theorem, which states that both values are equal, and their common value is the so-called average value, $V_{m}$, of a game on the unit square with kernel $F$.

It can be further shown (Karlin, 1957) that the number of support points of both $\mu_{x}^{*}$ and $\mu_{y}^{*}$ go to infinity as $k \rightarrow \infty$, that they are lower semicontinuous in $k$ and that they differ by at most 1 . Namely, the number of support points of $\mu_{x}^{*}$ is not less than that of $\mu_{y}^{*}$.

\section{References}

Apostol, T. M. (1974). Mathematical analysis. second ed., Reading, MA: Addison-Wesley.

Arimoto, S., \& Kimura, H. (1973). Optimal input test signals for system identification-an information theoretic approach. International Journal of Systems Science, 1(3), 279-290.

Atkinson, A. C., Chaloner, K., Juritz, J., \& Herzberg, A. M. (1993). Optimum experiment design for properties of a compartmental model. Biometrica, 29, 325-337.

Atkinson, A. C., \& Doner, A. N. (1992). Optimum experiment design. Oxford: Clarendon Press.

Bartle, R. G. (1966). The elements of integration. New York: Wiley.
Başar, T., \& Bernhard, P. (1995). $H^{\infty}$ _optimal control and related minmax design problems: a dynamic game approach. Boston-Basel, Berlin: Birkhauser.

Başar, T., \& Olsder, G. J. (1995). Dynamic noncooperative game theory. 2nd ed., London, New York: Academic Press.

Biedermann, S., \& Dette, H. (2003). A note on maximin and Bayesian $d$ optimal designs in weighted polynomial regression. Mathematical Methods of Statistics, 12(3), 358-370.

Bohnenblust, H. F., Karlin, S., \& Shapley, L.S., 1950. Games with a continuous, convex pay-off. In Contributions to the theory of games, Annals of mathematics studies. (Vol. I. No. 24, pp. 181-192). Cambridge: Princeton University Press.

Bombois, X., Scorletti, G., Gevers, M., Van den Hof, P., \& Hildebrand, R. (2005). Least costly identification experiment for control. Technical Report, Louvian-la-Neuve: CESAME.

Chaloner, K., \& Larntz, K. (1989). Optimal Bayesian designs applied to logistic regression experiments. Journal of Statistical Planning and Inference, 21, 191-208.

Chaloner, K., \& Verdinelli, I., 1995. Bayesian experiment design: A review. Statistical Science, 10(3), 273-304.

Chernoff, H. (1975). Approaches in sequential design of experiments. In J. Srivastava (Ed.), Survey of statistical design (pp. 67-90). Amsterdam: North-Holland

Cox, D. R. (1958). Planning of experiments. New York: Wiley.

D'Argenio, D. Z., \& Van Guilder, M., 1988. Design of experiments for parameter estimation involving uncertain systems, with application to pharmacokinetics. In 12th IMACS World congress on scientific computation (vol. 2, pp. 511-513).

Dantzig, G. B. (1951). A proof of the equivalence of the programming problem and the game problem. In T. C. Koopmans (Ed.), Activity analysis of production and allocation: Proceedings of a conference. 
Cowles commission for research in economics (pp. 330-335). New York Wiley.

Dette, H., Melas, V. B., \& Pepelyshev, A. (2003). Standardized maximin $e$ optimal designs for the Michaelis-Menten model. Statistica Sinica, 13(4), 1147-1163.

El-Gamal, M. A., \& Palfrey, T. R. (1996). Economical experiments: Bayesian efficient experimental design. International Journal of Game Theory, 25(4), 495-517.

Fedorov, V. V. (1972). Theory of optimal experiments. New York, London: Academic Press.

Fedorov, V. V. (1980). Convex design theory. Mathematische Operationsforschung und Statistik Series Statistics, 11(3), 403-413.

Ford, I., \& Silvey, S. D. (1980). A sequentially constructed design for estimating a nonlinear parametric function. Biometrika, 67(2), 381-388.

Ford, I., Titterington, D. M., \& Wu, C. F. J. (1985). Inference and sequential design. Biometrika, 72(3), 545-551.

Fudenberg, D., \& Tirole, J. (1991). Game Theory. Cambridge, MA: MIT Press.

Gagliardi, R. M. (1967). Input selection for parameter identification in discrete systems. IEEE Transactions on Automatic Control, AC-12(5), 133-147.

Gale, D., Kuhn, H. W., \& Tucker, A. W. (1951). Linear programming and the theory of games. In T. C. Koopmans (Ed.), Activity analysis of production and allocation: Proceedings of a conference. Cowles commission for research in economics (pp. 317-329). New York: Wiley.

Gevers, M. (2005). Identification for control: From the early achievements to the revival of experiment design. European Journal of Control, 11, 1-18.

Gevers, M., \& Bombois, X. (2006). Input design: From open-loop to control-oriented design. In 14th IFAC symposium on system identification (SYSID'06). Newcastle, Australia (pp. 1329-1334).

Glicksberg, I. L. (1950). Minimax theorem for upper and lower semicontinuous payoffs. Rand Corporation Research Memorandum RM478.

Goodwin, G. C., Graebe, S. F., \& Salgado, M. E. (2001). Control system design. Upper Saddle River, NJ: Prentice-Hall.

Goodwin, G. C., Murdoch, J. C., \& Payne, R. L. (1973). Optimal test signal design for linear single input-single output system identification. International Journal of Control, 17(1), 45-55.

Goodwin, G. C., \& Payne, R. L. (1973). Design and characterisation of optimal test signals for linear single input-single output parameter estimation. In third IFAC symposium, The Hague/Delft.

Goodwin, G. C., \& Payne, R. L. (1977). Dynamic system identification: Experiment design and data analysis. New York: Academic Press.

Goodwin, G. C., Payne, R. L., \& Murdoch, J. C. (1973). Optimal test signal design for linear single input-single output closed loop identification. In CACSD conference, Cambridge.

Goodwin, G. C., Welsh, J. S., Feuer, A., \& Derpich, M. (2006). Utilizing prior knowledge in robust optimal experiment design. In Proceedings of the 14th IFAC SYSID, Newcastle, Australia (pp. 1358-1363).

Hildebrand, R., \& Gevers, M. (2003a). Identification for control: Optimal input design with respect to a worst-case $v$-gap cost function. SIAM Journal of Control Optimization, 41(5), 1586-1608.

Hildebrand, R., \& Gevers, M. (2003b). Minimizing the worst-case $v$-gap by optimal input design. In Proceedings of the 13th IFAC symposium on system identification (pp. 665-670).

Hjalmarsson, H. (2005). From experiment design to closed-loop control. Automatica, 41(3), 393-438.

Horn, R. A., \& Johnson, C. R. (1985). Matrix analysis. Cambridge: Cambridge University Press.

Karlin, S. (1957). On games described by bell shaped kernels. In Contributions to the theory of games. Annals of mathematics studies (Vol. III. No. 39, pp. 365-391). New Jersey: Princeton University Press.

Karlin, S., \& Studden, W. J. (1966). Optimal experimental designs. Annals of Mathematical Statistics, 37, 783-815.
Kempthorne, O. (1952). Design and analysis of experiments. New York: Wiley. Kiefer, J. (1974). General equivalence theory for optimum designs (approximate theory). Annals of Statistics, 2(5), 849-879.

Kiefer, J., \& Wolfowitz, J. (1960). The equivalence of two extremum problems. Canadian Journal of Mathematics, 12, 363-366.

Krattenthaler, C. (1998). Advanced determinant calculus. Séminaire Lotharingien de Combinatoire, Vol. 42, 67pp.

Landaw, E. M. (1984). Robust sampling designs for compartmental models under large prior eigenvalue uncertainties. In J. Eisenfeld, \& C. DeLisi (Eds.), Mathematics and computers in biomedical applications (pp. 181 -187). Amsterdam, North-Holland: Elsevier.

Levadi, V. S. (1966). Design of input signals for parameter estimation. IEEE Transactions on Automatic Control, AC-11(2), 205-211.

Mareels, I. M. Y., Bitmead, R. R., Gevers, M., Johnson, C. R., Kosut, R. L., \& Poubelle, M. A. (1987). How exciting can a signal really be? Systems \& Control Letters, 8, 197-204.

Mårtensson, J., \& Hjalmarsson, H. (2006). Robust input design using sum of squares constraints. In 14th IFAC symposium on system identification (SYSID'06), Newcastle, Australia (pp. 1352-1357).

McKinsey, J. C. C. (1952). Introduction to the theory of games. New York: McGraw-Hill.

Mehra, R. K. (1974). Optimal inputs for system identification. IEEE Transactions on Automatic Control, AC-19, 192-200.

Melas, V. B. (1978). Optimal designs for exponential regression. Mathematische Operationsforschung und Statistik Series Statistics, 9(1), 45-59.

Müller, W. G., \& Pötscher, B. M. (1992). Batch sequential design for a nonlinear estimation problem. In V. Fedorov, W. Müller, \& I. Vuchkov (Eds.), Model-orientated data analysis a survey of recent methods (pp. 77-87). Heidelberg: Physica-Verlag.

Owen, G. (1968). Game theory. Philadelphia: W.B. Saunders.

Owen, G. (1995). Game theory. 3rd ed., San Diego, CA: Academic Press.

Pronzato, L., \& Walter, E. (1988). Robust experiment design via maxmin optimisation. Mathematical Biosciences, 89, 161-176.

Rudin, W. (1973). Functional analysis. New York: McGraw-Hill.

Rudin, W. (1987). Real and complex analysis. 3rd ed., Singapore: McGrawHill.

Sebastiani, P., \& Wynn, H. P. (2000). Maximum entropy sampling and optimal Bayesian experimental designs. Journal of Royal Statistical Society: Series B, 62(1), 145-157.

Shimizu, K., \& Aiyoshi, E. (1980). Necessary conditions for min-max problems algorithms by a relaxation procedure. IEEE Transactions on Automatic Control, 25(1), 62-66.

Szép, J., \& Forgó, F. (1985). Introduction to the theory of games. Budapest, Hungary: D. Reidel Publishing Company.

Wald, A. (1943). On the efficient design of statistical investigations. Annals of Mathematical Statistics, 14, 134-140.

Walter, E., \& Pronzato, L. (1997). Identification of parametric models from experimental data. Berlin, Heidelberg, New York: Springer.

Welsh, J. S., Goodwin, G. C., \& Feuer, A. (2006). Evaluation and comparison of robust optimal experiment design criteria. In Accepted to the American control conference, Minneapolis, USA (http://www.eecs.newcastle.edu.au/ reports/EE05044.pdf).

Whittle, P. (1973). Some general points in the theory of optimal experimental design. Journal of Royal Statistical Society, 1, 123-130.

Wu, C. F. J. (1985). Asymptotic inference from sequential design in a nonlinear situation. Biometrika, 72(3), 553-558.

Wynn, H. P. (1972). Results in the theory and construction of $d$-optimum experimental designs. Journal of Royal Statistical Society, 2, 133-147.

Zarrop, M. (1979). Optimal experiment design for dynamic system identification. Lecture notes in control and information (vol. 21). Berlin, New York: Springer.

Zhou, K., Doyle, J. C., \& Glover, K. (1996). Robust and optimal control. Englewood Cliffs, NJ: Prentice-Hall. 


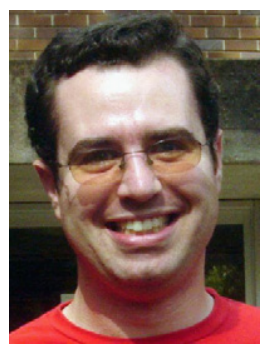

Cristian R. Rojas was born in 1980 . He received the professional title of Ingeniero Civil Electrónico and the M.S. degree in electronics engineering from the Universidad Técnica Federico Santa María, Valparaíso, Chile, in 2004, receiving the AEXA Award to the best graduated student. He is currently working toward the Ph.D. degree in electrical engineering at The University of Newcastle, NSW, Australia. His research interests include system identification, game theory, stochastic programming and Bayesian statistics.

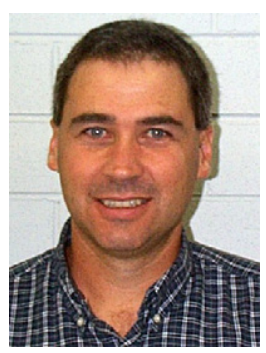

James Welsh was born in Maitland, Australia, in 1965. He received the B.E. degree (Hons. I) in electrical engineering from The University of Newcastle, Callaghan, NSW, Australia, in 1997. Dr. Welsh received his Ph.D. in 2004, which studied ill-conditioning problems arising in system identification, from the same university. $\mathrm{He}$ gained industrial experience from 1981 to 1997 with Bloomfield Collieries, AES, and TYP Pty. Ltd., Australia. During the last several years, he has been actively involved in research projects which include, Powertrain Control, Model Predictive Control and System Identification Toolboxes, with the Centre for Complex Dynamic Systems and Control, the University of Newcastle. His research interests include auto-tuning, system identification, and process control. Dr. Welsh is currently employed as a Lecturer in the School of Electrical Engineering and Computer Science at the University of Newcastle. He was awarded the University of Newcastle, Faculty of Engineering and Built Environment Prize for the Best International Journal Paper published by a postgraduate in 2003. In 1997, he won the Institute of Engineers Australia National Undergraduate Thesis Prize in Automation, Control, and Instrumentation.

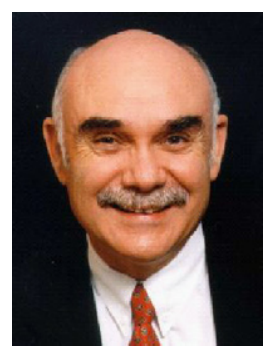

Graham Goodwin obtained a B.Sc (Physics), B.E. (Electrical Engineering), and Ph.D from the University of New South Wales. He holds Honorary Doctorates from Lund Institute of Technology, Sweden and the Technion Israel. From 1970 until 1974 he was a lecturer in the Department of Computing and Control, Imperial College, London. Since 1974 he has been with The University of Newcastle, Australia where he is currently Professor of Electrical Engineering. $\mathrm{He}$ is the co-author of eight monographs: Control
Theory, Oliver and Boyd (1970), Dynamic System Identification, Academic Press (1977), Adaptive Filtering, Prediction and Control, Prentice Hall (1984), Digital Control and Estimation, Prentice Hall (1989), Sampling in Digital Signal Processing and Control, Birkhauser (1996), Fundamental Limitations in Filtering and Control, Springer (1997), Control System Design, Prentice Hall (2001), Constrained Control and Estimation, Springer (2004). He is also the author of four edited volumes, and many technical papers. Graham is the recipient of Control Systems Society 1999 Hendrik Bode Lecture Prize, a Best Paper award by IEEE Trans. Automatic Control, a Best Paper award by Asian Journal of Control, and two Best Engineering Text Book awards from the International Federation of Automatic Control. Graham is a Fellow of IEEE; an Honorary Fellow of Institute of Engineers, Australia; a Fellow of the Australian Academy of Science; a Fellow of the Australian Academy of Technology, Science and Engineering; a Member of the International Statistical Institute; a Fellow of the Royal Society, London and a Foreign Member of the Royal Swedish Academy of Sciences.

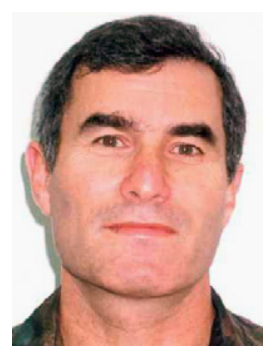

Arie Feuer received a B.Sc. and M.Sc. in Mechanical engineering from the Technion - Israel Institute of Technology, Haifa, Israel (1967 and 1973, respectively.) and a Ph.D. from Yale University in 1978 . From 1967 to 1970 he worked for Technomatics Inc. where he was involved in the design of automatic machines. During the period from 1978 through to 1983 Professor Feuer worked for Bell Labs on network performance evaluation. In 1983 he joined the faculty of Electrical Engineering at the Technion where he is currently a professor and head of the Control and Robotics Laboratory. Current research interests include: resolution enhancement of digital images and videos, sampling and combined representations of signals and images, and adaptive systems in signal processing and control. 